\title{
Nonprostatic diseases on PSMA PET imaging: a spectrum of benign and malignant findings
}

\author{
Felipe de Galiza Barbosa, Marcelo Araujo Queiroz * (D), Rafael Fernandes Nunes, Larissa Bastos Costa, \\ Elaine Caroline Zaniboni, José Flavio Gomes Marin, Giovanni Guido Cerri and Carlos Alberto Buchpiguel
}

\begin{abstract}
PSMA PET imaging was originally used to assess biochemical recurrence of prostate cancer (PCa), but its clinical use was promptly extended to detection, staging and therapy response assessment. The expanding use of PSMA PET worldwide has also revealed PSMA ligand uptake in diverse nonprostatic diseases, which raised questions about the specificity of this imaging modality. Although not very common initially, a growing number of pathologies presenting PSMA uptake on PET have been reported in the last few years, and a proper interpretation of PSMA PET imaging findings suddenly became challenging and, to some extent, confusing. Compared to cytoplasmic PSMA expression in nonprostatic cells, the molecular features of apical PSMA expression in PCa cells can help to distinguish these various conditions. Correlations of imaging findings to patient history, to the expected pattern of disease spread and mainly to computed tomography (CT) and/or magnetic resonance imaging (MRI) characteristics will reinforce the distinction of lesions that are more likely related to PCa from those that could lead to an incorrect diagnosis. The overall benefits of endothelial PSMA expression, which is associated with the neovasculature of malignant neoplasms, will be highlighted, stating the potential use of PSMA ligand uptake as a theranostic tool. This review aims to cover the collection of nonprostatic diseases, including benign and malignant tumors, in a didactic approach according to disease etiology, with discussion of bone-related conditions and inflammatory and infectious processes.
\end{abstract}

Keywords: Prostate cancer, Positron emission tomography, (68)Ga-PSMA

\section{Background}

Prostate cancer ( $\mathrm{PCa})$ management has been revolutionized with the advent of prostate-specific membrane antigen (PSMA) positron emission tomography (PET) imaging. Accordingly, the literature has been flooded with an enormous diversity of cases of incidentally detected PSMA uptake in nonprostatic conditions, which led to questioning the specificity of PSMA PET results $[1,2]$. However, a recent meta-analysis including 4790 patients who underwent ${ }^{68} \mathrm{Ga}$-PSMA PET/CT for

\footnotetext{
* Correspondence: marcelo.queiroz@hc.fm.usp.br

Department of Radiology, Hospital Sirio-Libanes, Rua Dona Adma Jafet, 91, Sao Paulo ZIP: 01308-050, Brazil
}

different indications showed a per-lesion specificity of 99\% [3]. Therefore, the nonprostatic diseases that exhibit PSMA uptake on PET imaging represent an uncommon finding that need to be recognized not only to avoid misdiagnosis but also to allow for correct therapy planning. On the other hand, although nonprostatic PSMA uptake might hamper the diagnostic performance of PSMA PET imaging, the receptor expression observed in some malignant tumor cells broadens the application of its use in diagnosis or even therapeutic approaches using PSMA-targeted radionuclide therapy [4]. Since it is a new imaging modality, even the normal biodistribution of PSMA on PET imaging is often revisited, and other 
anatomical sites are related as normal variants. Intense PSMA uptake is usually observed in lacrimal, parotid and submandibular glands, and in the small intestine, kidneys, liver, spleen and bladder, while mild to moderate uptake may be observed in nasal and esophageal mucosa; the vocal cords, gallbladder and biliary tract; tracheal and proximal bronchi; mediastinal, axillary and inguinal lymph nodes; gynecomastia; and sympathetic ganglia, such as stellate, celiac, hypogastric and presacral [5-7]. An overview of these conditions with physiological and benign PSMA uptake is shown on Figs. 1 and 2.

In this review, the nonprostatic conditions exhibiting PSMA uptake were divided in a didactic manner according to the underlying etiologies of the diseases: bonerelated conditions, inflammatory and infectious processes (including surgery-related findings) and benign and malignant tumors. It is important to note that all of our patients underwent PET imaging with ${ }^{68} \mathrm{Ga}$-PSMA-
11 (herein referred to as only PSMA) as the PET tracer, but the rationale of nonprostatic uptake is likely transferable to all PSMA-based available PET tracers (e.g., ${ }^{68} \mathrm{Ga}$-PSMA-I\&T, ${ }^{68} \mathrm{Ga}$-THP-PSMA ${ }^{18} \mathrm{~F}$-DCFPyL, and ${ }^{18}$ F-PSMA-1007), based on the biological mechanism of PSMA uptake hereinafter detailed. The key features for differential diagnosis of metastatic diseases will also be thoroughly highlighted to warrant an appropriate imaging interpretation.

\section{Main text}

\section{Mechanism of PSMA uptake}

PSMA is a type II (i.e., integral) transmembrane glycoprotein that was discovered in 1987 in metastatic $\mathrm{PCa}$ cell lines [8]. PSMA is encoded by the FOLH1 gene in the short arm of chromosome 11 and is formed by 750 amino acids (aa), divided into intracellular, transmembrane and extracellular regions. The last region is the largest, with 707 aa, and contains specific enzymatic

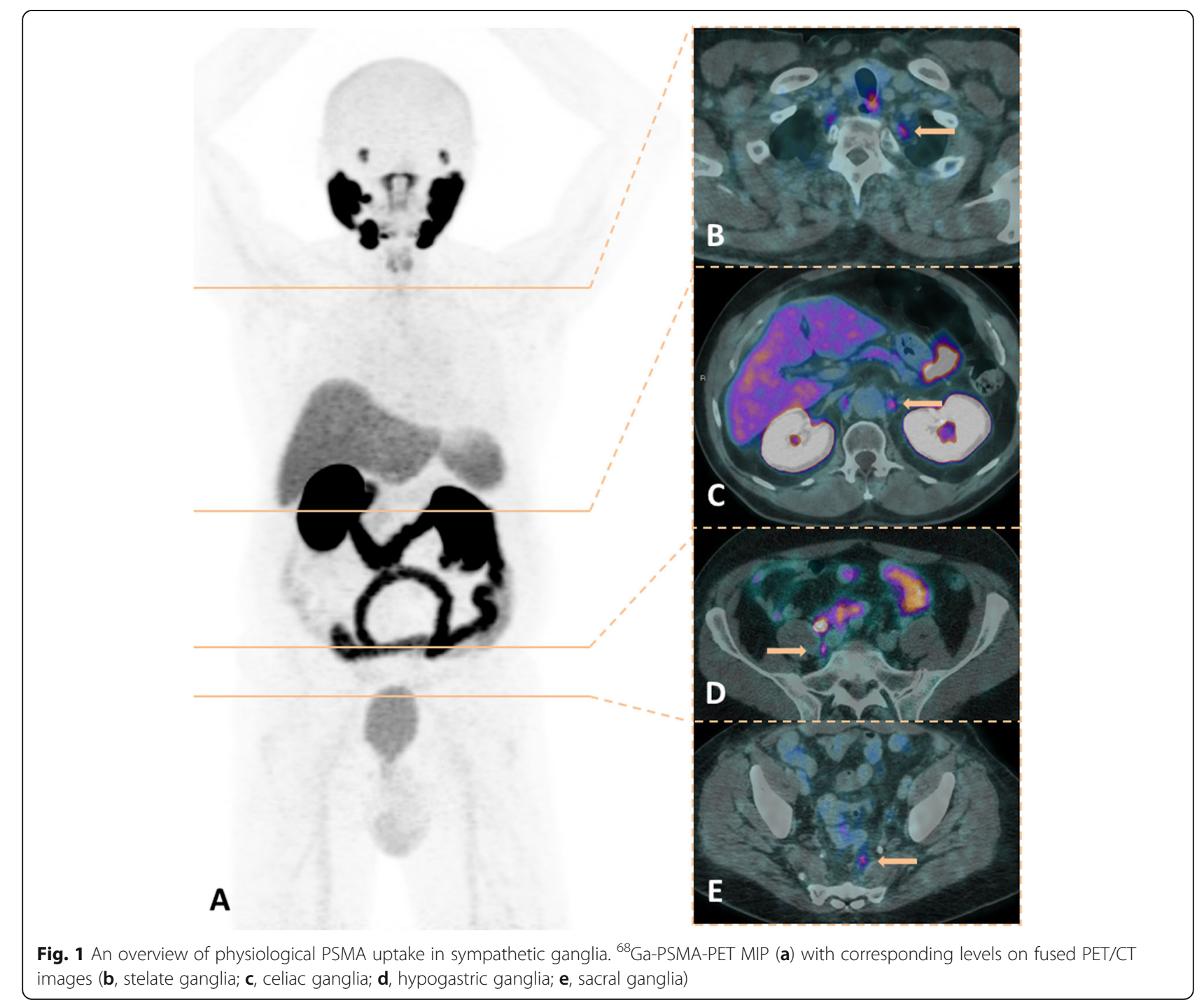




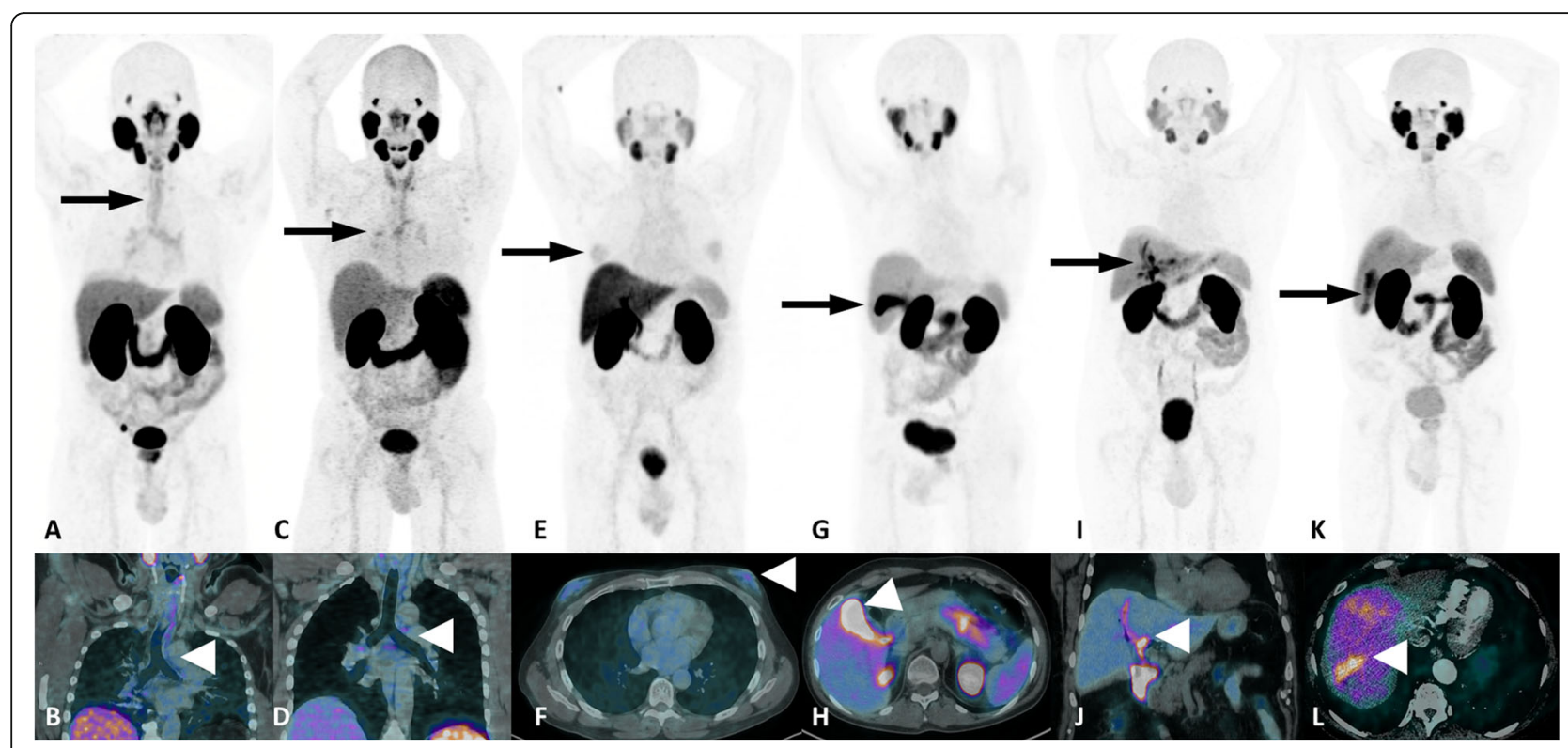

Fig. 2 A miscelanea of normal variants of PSMA uptake. ${ }^{68} \mathrm{Ga}-\mathrm{PSMA-PET} \mathrm{MIP}$ images on top (a, $\mathbf{c}, \mathbf{e}, \mathbf{g}, \mathbf{i}$ and $\left.\mathbf{k}\right)$ and corresponding fused PET/CT

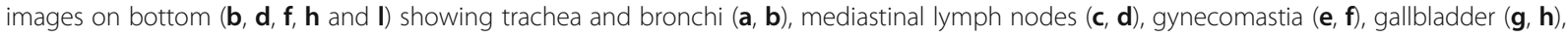
billiary tract $(\mathbf{i}, \mathbf{j})$ and liver perfusion defect $(\mathbf{k}, \mathbf{I})$

domains, which are the main target for current PSMAligand imaging and therapy [9]. In contrast with other clinically relevant prostate-related antigens, such as prostate-specific antigen or prostatic acid phosphatase, which are secretory proteins, the PSMA transmembrane conformational structure enables it to exhibit an internalization functionality by means of endosomal complexes, which is a highly attractive feature for targeted diagnostic and therapeutic approaches, especially with radiopharmaceuticals $[10,11]$. PSMA functions are not completely understood, but reported functions include enzymatic peptidase activity related to folate and glutamate metabolism, as well as activation of signaling pathways (e.g., Akt and MAPK) involved in cell proliferation and survival [12]. PSMA expression occurs in normal epithelial prostate cells and is highly upregulated in PCa cells according to the biological aggressiveness of the disease, generally becoming more intense in high Gleason score, poorly differentiated, castration-resistant and metastatic tumors. Despite this well-known association, the exact role of PSMA in PCa cell differentiation status, proliferation and metastatic potential remains unclear [13].

However, in contrast to the idea induced by its name, PSMA expression is not exclusive to prostate cells and can be found in several other tissues and/or conditions, including normal nonprostatic epithelial cells, inflammation/infection, nonprostatic neoplastic cells and nonprostatic tumor-associated neovasculature. To better understand this variability in PSMA expression and its potential consequences on PSMA-based image interpretation, the following concepts should be kept in mind by readers.

\section{Apical membrane vs cytoplasmic PSMA expression}

From the structural perspective of an epithelial cell, PSMA expression may occur in the apical (i.e., luminal) membrane, in the cytoplasm or in both. Apical membrane expression is the prototype of PSMA expression, occurring in normal prostatic epithelial cells, and mostly in PCa cells (Fig. 3a). Apical expression is markedly amplified in PCa cells in comparison with normal prostatic epithelial cells and other tissues/neoplastic cells. This type of expression leads to intense PSMA accumulation on both immunohistochemistry and imaging studies. A study of PSMA expression with immunohistochemistry in 3161 varied tumors found that apical membrane expression tends to be more intense and occurs only in PCa because all of the nonprostatic tumors exhibited PSMA expression in the cytoplasm, which is normally less intense than that in the membrane [14]. Interestingly, Osman and colleagues, who studied synchronic malignancies that were detected in a cohort of 764 patients that underwent PSMA PET, reached similar findings some years later. A higher uptake (despite some overlap, especially in lung lesions) in PCa lesions confirms higher specificity of PSMA overexpression to detect PCa lesions, which was likely an observation of the same phenomena in the light of a new technology (7). More recently, Rischpler et al recalled this theme when studying PSMA ligand uptake patterns in sympathetic ganglia and showed consistently lower 

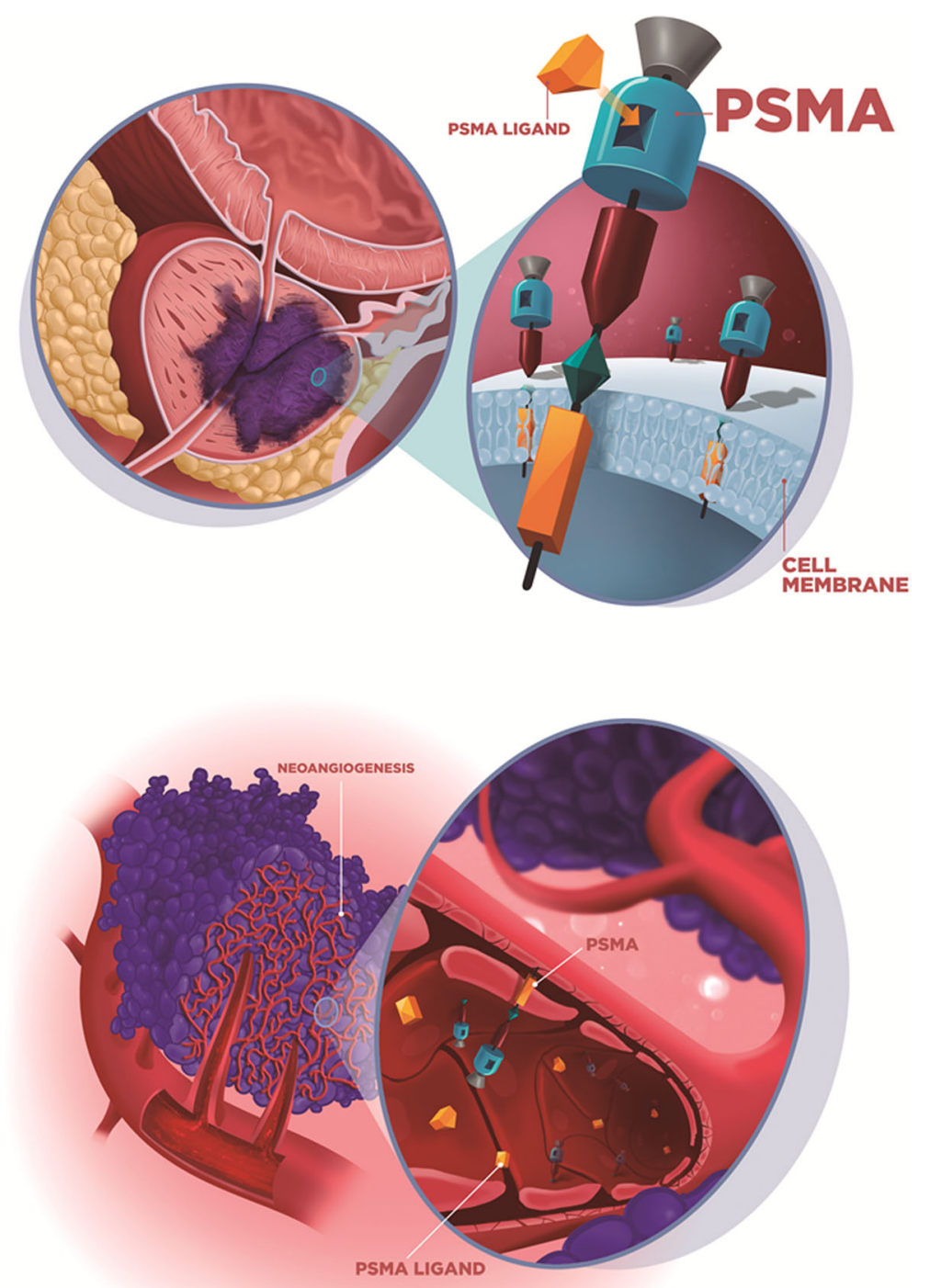

Fig. 3 llustration of the two main mechanism os PSMA expression that explains the radiotracer uptake in prostate cancer (a) and other nonprostatic causes with endothelial expression (b)

PSMA ligand uptake in these structures than in with PCa metastatic lesions [15]. Despite the unquestionable consistency of this finding across several studies, no validated threshold of PSMA ligand uptake was found until now to safely differentiate PCa from nonprostatic lesions, highlighting the importance of recognizing morphological and biological patterns of different diseases to produce clinically valuable reports.

\section{Endothelial expression}

Endothelial PSMA expression has been extensively studied and occurs in almost all nonprostatic solid tumors associated with neovasculature but not in benign endothelial tissue, raising some hypotheses about the role of
PSMA in neoangiogenesis and vascular growth factors regulation $[12,16]$ (Fig. 3b). Therefore, PSMA ligand uptake by nonprostatic solid tumors (benign and malignant) in PET imaging must be interpreted with this knowledge to help readers pay attention to histological, imaging and clinical information about tumor neovascularization. Important remarks are the lack of PSMA expression in the endothelial cells of normal tissues and, interestingly, in $\mathrm{PCa}$-associated neovasculature.

\section{Inflammation/infection expression}

Few data are available about the mechanism of PSMA expression by immune cells. It is possible that neovascularization also plays a role in this scenario as well as an 
increased availability of PSMA ligand to the site of inflammation/infection due to an increase in regional blood flow/vascular permeability. Additionally, some inference about macrophage folate receptors and their potential implications in PSMA ligand imaging has been suggested [17]. However, in the PSMA ligand PET era, an increasing number of reports of false-positive findings in inflammatory/infectious diseases have been published, making this a practical question that requires the attention of readers in daily reporting.

\section{Nonprostatic diseases}

Lesion evaluation can be challenging in specific situations, and practitioners should use a combination of aspects: a) molecular (i.e., are the intensity, extension, and semiquantitative patterns different from those of other lesions or from those expected to occur in $\mathrm{PCa}$ ?); b) morphological (i.e., are the CT/MRI findings typical of a specific neoplasm or of a PCa lesion?); c) biological (i.e., does this lesion reasonably fit with known PCa staging, predicted spread, and/or PSA values?); and d) clinical (i.e., is there some underlying condition/comorbidity that can be linked to this lesion?). Of course, there is no absolute discriminative answer generated by these questions, but available data about patterns of uptake (tends to be much higher in $\mathrm{PCa}$, despite some potential overlap in organs such as the lungs or liver), knowledge of CT/MRI morphological disease patterns and the understanding of the natural history of various neoplasms allow interpreters to produce more accurate predictions, for example, indicating biopsies with more effectiveness. A list including all the reported cases of nonprostatic diseases with PSMA ligand uptake on PET imaging is summarized in Table $1[4,17-32]$.

\section{Bone-related conditions}

Bone is the most common site of metastatic hematogenous spread in PCa patients. PSMA PET is considered the most accurate and sensitive method not only to evaluate skeletal involvement [33], but also to possibly differentiate ambiguous finding and diagnose benign pathologies.

There are no specific features of PCa bone metastases, but it customarily appears in a high-risk PCa patient presenting multiple blastic lesions predominantly in the pelvis and spine due to the particularities of bone spreading [34]. PSMA avidity has a tendency of being higher in regard to appraising malignant bone lesions in contrast to benign pathologies, which usually present low to moderate uptake. Castrate-resistant prostate cancer patients tend to have metastases with higher PSMA expression compared to those with hormone-sensitive cancer. Therefore, in this specific clinical setup, it is even more likely to expect high PSMA avidity on PCa bone lesions.

The morphological aspects on CT, such as the number of lesions, type of margin, pattern of bone destruction, type of periosteal reaction and presence of an associated soft tissue mass, should always be taken into consideration when evaluating a bone lesion. A benign appearance customarily presents as a slow-growing lesion with geographic destruction, sharply demarcated margins and smooth and uninterrupted periosteal reaction without soft tissue mass.

Osteodegenerative changes are very common among PCa patients, but only very few of them may present mild PSMA uptake, especially in the spine. The clue for diagnosis is the typical morphological appearance of joint space narrowing with sclerosis and osteophytosis.

Another very common finding on PSMA PET imaging is a single mild tracer uptake in the ribs (Fig. 4). It is usually not related to $\mathrm{PCa}$ and does not require further investigation (i.e. bone scan or MRI) as the dedicated CT from PET/CT is often sufficient for a confident diagnosis. In few unclear cases a dedicated thoracic CT with a bone filter should offer a better morphological correlation, such as small fibrous osseous defect and a tiny fracture. It is also important to apply a close correlation to the patient's clinical history, for instance, in cases of recent trauma in the thorax.

PSMA uptake in osteomyelitis might be related to transient vascular distress in a patient with a typical clinical history (systemic inflammation, past trauma or ulcer). In these cases, it is mandatory to correlate PET findings with CT (inhomogeneous osteosclerosis with bony sequestrum) and/or MRI (low T1 signal within the bone), indicating cortical destruction and bone marrow edema. Abscess and soft tissue collections may be observed after injection of gadolinium (Fig. 5).

A single lesion often favors a benign finding, e.g., fibrous dysplasia, which appears as an expansive welldefined lesion with ground-glass attenuation. Benign lesions can also appear as focal mild medullary uptake without any correlated structural bone lesions on CT. On the other hand, PCa metastasis without CT correspondence appears as moderate to high PSMA focal uptake in the bone marrow, often multiple, which is more common in very aggressive disease when the bone destruction has not been demonstrated yet [35]. Faint and heterogeneous PSMA uptake delineating the cortex of pelvic bones is associated with characteristic morphological changes, such as diffuse sclerosis, thickened cortex, bone expansion, and coarsened trabeculae, and is typical for Paget's disease. See Fig. 6.

Despite the fact that multiple lesions are likely to have a secondary origin, we still need to pay close attention to the morphologic aspects (lytic vs blastic lesions) and 
Table 1 A list of published case reports of nonprostatic diseases presenting PSMA uptke according to the etiology

\begin{tabular}{|c|c|c|c|c|}
\hline & $\begin{array}{l}\text { Bone-related } \\
\text { conditions }\end{array}$ & $\begin{array}{l}\text { Inflammatory and } \\
\text { infectious processes }\end{array}$ & Benign neoplasms & Malignant neoplasms \\
\hline $\begin{array}{l}\text { Central and peripheral } \\
\text { nervous system }\end{array}$ & - & Neurocysticercosis & $\begin{array}{l}\text { Meningioma } \\
\text { Schwannoma } \\
\text { Peripheral nerve sheath tumor } \\
\text { Neurofibromas }\end{array}$ & Gliomas \\
\hline Cervical & - & - & Thyroid and parathyroid adenomas & $\begin{array}{l}\text { Head and neck Squamous } \\
\text { cell carcinoma } \\
\text { Adenoid cystic carcinoma } \\
\text { Thyroid cancer }\end{array}$ \\
\hline Thoracic & - & $\begin{array}{l}\text { Opacities and bronchietasis } \\
\text { Sarcoidosis } \\
\text { Tuberculosis } \\
\text { Anthracosilicosis } \\
\text { Berylliosis }\end{array}$ & $\begin{array}{l}\text { Elastofibroma dorsi } \\
\text { Pseudoangiomatous stromal } \\
\text { hyperplasia of the breast } \\
\text { Thymoma }\end{array}$ & $\begin{array}{l}\text { Breast cancer } \\
\text { Lung cancer } \\
\text { Thymoma } \\
\text { Mesothelioma }\end{array}$ \\
\hline Abdominal & - & $\begin{array}{l}\text { Postsurgical inflammation } \\
\text { Liver and splenic } \\
\text { sarcoidosis } \\
\text { Diverticulosis } \\
\text { Amyloidosis of seminal } \\
\text { vesicles } \\
\text { Anal fistula }\end{array}$ & $\begin{array}{l}\text { Angiolipoma } \\
\text { Hepatic and splenic hemangiomas } \\
\text { Adrenal adenoma } \\
\text { Pancreatic serous cystadenoma }\end{array}$ & $\begin{array}{l}\text { Hepatocellular carcinoma } \\
\text { Cholangiocarcinoma } \\
\text { Adrenocortical carcinoma } \\
\text { Renal cell carcinoma } \\
\text { Transitional cell carcinoma } \\
\text { Pancreatic carcinoma } \\
\text { Neuroendocrine tumor } \\
\text { Gastric GIST } \\
\text { Gastric adenocarcinoma } \\
\text { Bladder Paraganglioma } \\
\text { Cervical carcinoma } \\
\text { Ovary cancer } \\
\text { Endometrium cancer } \\
\text { Vulvar carcinoma }\end{array}$ \\
\hline $\begin{array}{l}\text { Skeletal, soft-tissues and } \\
\text { vascular }\end{array}$ & $\begin{array}{l}\text { Osteomyelitis } \\
\text { Fracture } \\
\text { Paget's Disease } \\
\text { Hemangioma } \\
\text { Osteogenerative } \\
\text { Fibrous Osseous } \\
\text { Defect } \\
\text { Fibrous Dysplasia } \\
\text { Sacral insufficiency } \\
\text { after RT } \\
\text { Osteochondroma } \\
\text { Multiple Myeloma } \\
\text { Polycythemia } \\
\text { Rubra Vera } \\
\text { Myelodysplasia }\end{array}$ & Nodular fasciitis & $\begin{array}{l}\text { Dermatofibroma } \\
\text { Acrochordon } \\
\text { Fibromatosis } \\
\text { Desmoid tumor } \\
\text { Intramuscular myxoma } \\
\text { Hemangiopericytoma } \\
\text { Nasal Angiofibroma } \\
\text { Angiolipoma } \\
\text { Acrochordon } \\
\text { Fibromatosis }\end{array}$ & $\begin{array}{l}\text { Lymphoma } \\
\text { Osteosarcoma } \\
\text { Ewing sarcoma } \\
\text { Melanoma } \\
\text { Malignant nerve sheath } \\
\text { tumor } \\
\text { Multiple Myeloma } \\
\text { Other soft tissue sarcomas }\end{array}$ \\
\hline
\end{tabular}

notably to other exams (imaging and laboratory findings) in addition to clinical history, as there are known underlying comorbidities (Fig. 7). In contrast, diffuse, faint and homogeneous uptake in the bone marrow is most likely related to an underlying hematological disease rather than to neoplastic infiltration, which more often appears with heterogeneous and intense PSMA uptake (Fig. 8).

Although low to moderate PSMA avidity is expected when evaluating nonprostatic bone-related diseases, some lesions might appear with higher uptake, which could lead to an erroneous diagnosis (metastases), particularly in studies without morphological correlation, for which further investigation (MRI and/or biopsy) is mandatory. PSMA uptake in bone hemangiomas is variable, and typical CT appearance is essential for diagnosis. The most common morphological finding is thickened vertical trabeculae lesions surrounded by fat marrow or vascular lacunae presenting a faint PSMA uptake. Occasionally, the morphological aspects on CT are not typical or even absent, and in those cases, proceeding with a different type of morphological exam, such as MRI, would be advised. Hemangiomas with high uptake usually present PSMA expression in the endothelial cells, and when morphological findings are not enough to determine a diagnosis, a biopsy should be the next step. See Fig. 9.

\section{Inflammatory and infectious processes}

Among various inflammatory and infectious processes, PSMA uptake not related to prostate cancer has already been described in numerous thoracic, abdominal and musculoskeletal conditions. Additionally, an 


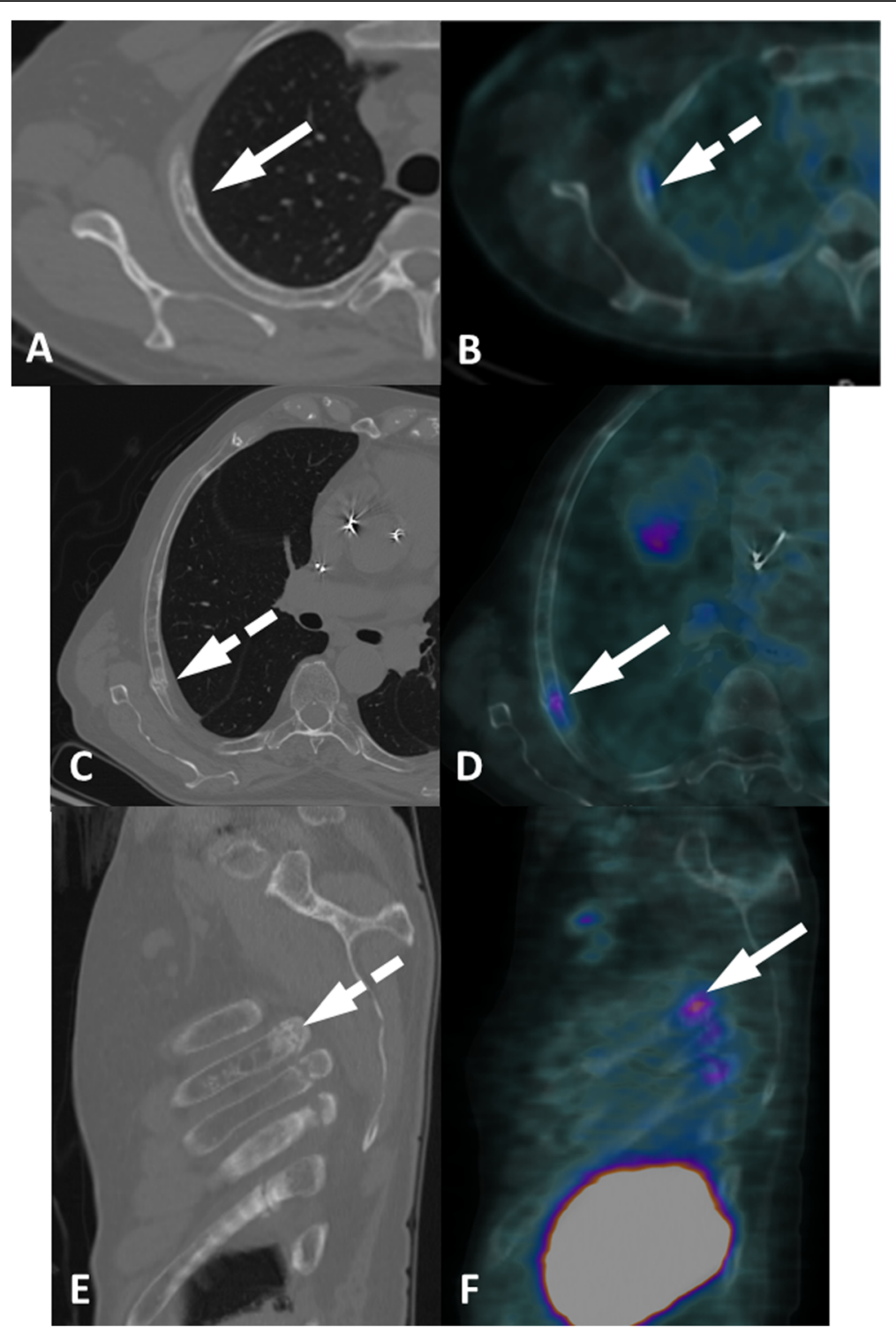

Fig. 4 Focal PSMA uptake in the ribs in two different patients. Patient 1, fibrous osseus defect (a, b). Axial CT (a) and axial ${ }^{68} \mathrm{Ga}-\mathrm{PSMA}$-PET/CT (b) show a single focus of faint uptake (dashed arrow) in a typical fibrous osseous defect of the third right rib (arrow). Patient 2, ribs fractures (c-f). Axial CT (c), sagittal CT (e), axial ${ }^{68} \mathrm{Ga}-\mathrm{PSMA}-\mathrm{PET} / \mathrm{CT}$ (d) and sagittal ${ }^{68} \mathrm{Ga}-\mathrm{PSMA}-\mathrm{PET} / \mathrm{CT}$ (f) show mild uptake (arrows) in continuous ribs fractures (dashed arrows)

inflammatory PSMA uptake in the prostatic bed might occur following radical prostatectomy, and also after other surgeries.

The thoracic manifestations of prostate cancer, apart from bone involvement of the vertebral bodies, ribs, sternum, scapulae and clavicles, are mostly confined to the lungs and pleura. Metastases to the lungs are often multiple solid, rounded nodules of varying size that occur along with other metastatic sites (usually skeletal), while the pleural involvement of $\mathrm{PCa}$ is represented by diffuse, irregular or nodular thickening of the pleura. On the other hand, inflammatory and infectious processes occur more common in the lungs, are morphologically different and have minimal PSMA uptake. On CT, they range from single solid nodules, often irregular or surrounded by ground glass opacities, to pulmonary consolidations and atelectasis, both associated with reactive lymph nodes in the hilum and mediastinum or bronchiectasis. Interstitial and granulomatous diseases may also present mild PSMA uptake, such as usual interstitial fibrosis, sarcoidosis and tuberculosis. See Fig. 10. The morphological findings on CT allow for differentiation from an eventual and rare form of lymphangitic carcinomatosis related to PCa. Occupational lung diseases such as 

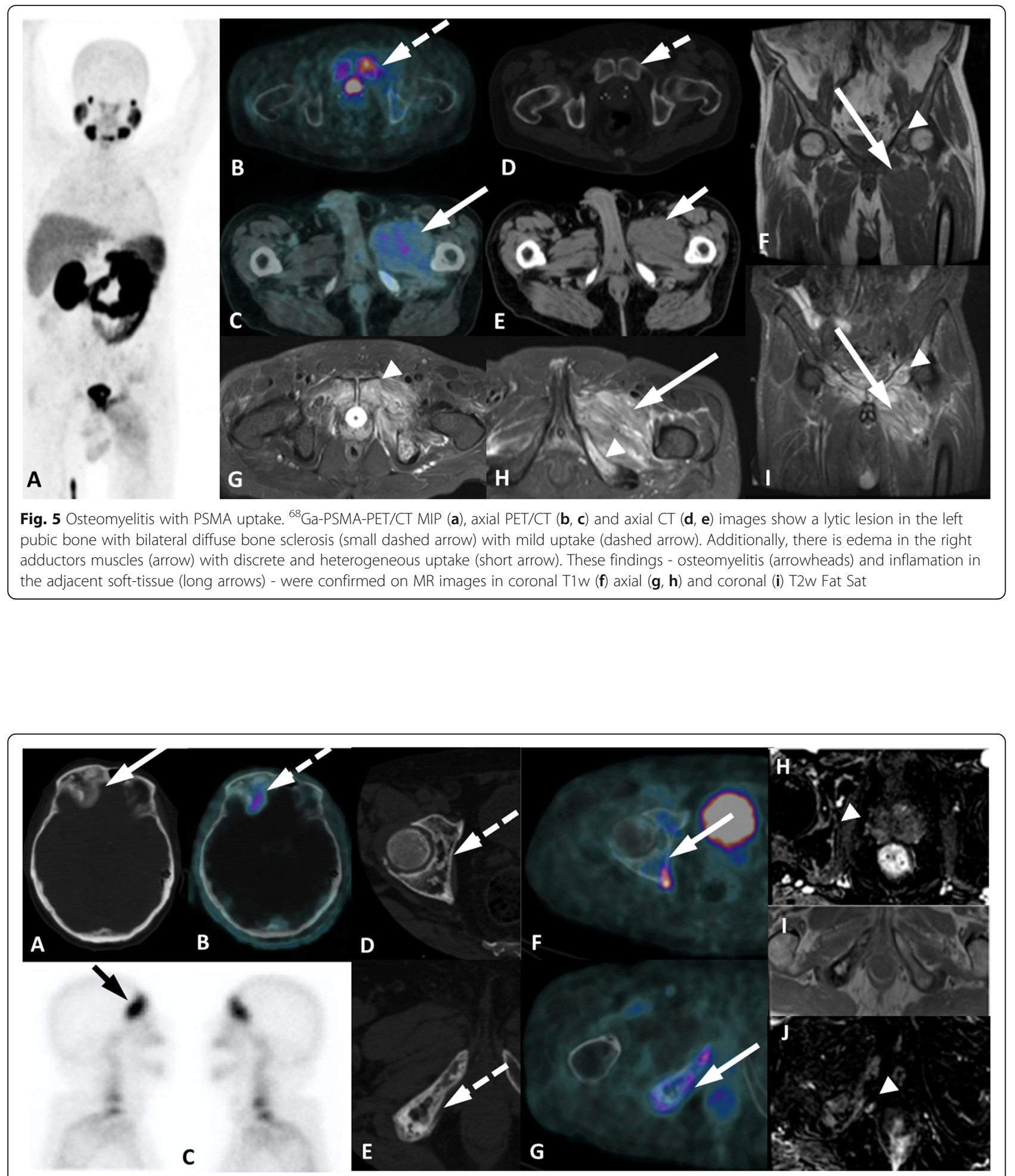

Fig. 6 Bone PSMA uptake in correlation with morphology and other imaging modalities in two different patients. Patient 1, fibrous dysplasia (ac). Axial CT (a) and axial ${ }^{68} \mathrm{Ga}$-PSMA-PET/CT (b) show a homogeneously sclerotic lesion with well-defined borders (arrow) and mild PSMA uptake (dashed arrow), suggestive of fibrous dysplasia. Previous MDP-bone scan lateral images (c) showed an intense osteogenic reaction (black arrow) in the right frontal bone, which is impossible to differentiate from a bone metastatis without a CT. Patient 2, Paget's disease (d-j). Axial CT (d, e), axial ${ }^{68} \mathrm{Ga}$-PSMA-PET/CT (f, $\mathbf{g}$ ) and axial T1w MR (i) images show mild uptake (arrows) in the cortical thickening and sclerosis of the right ischium (dashed arrows). Axial perfusion ( $\mathbf{h}, \mathbf{j})$ MR images show an increased vascularization exactly in the same areas of the PSMA uptake (arrowheads). These findings are highly suggestive of Paget's disease 


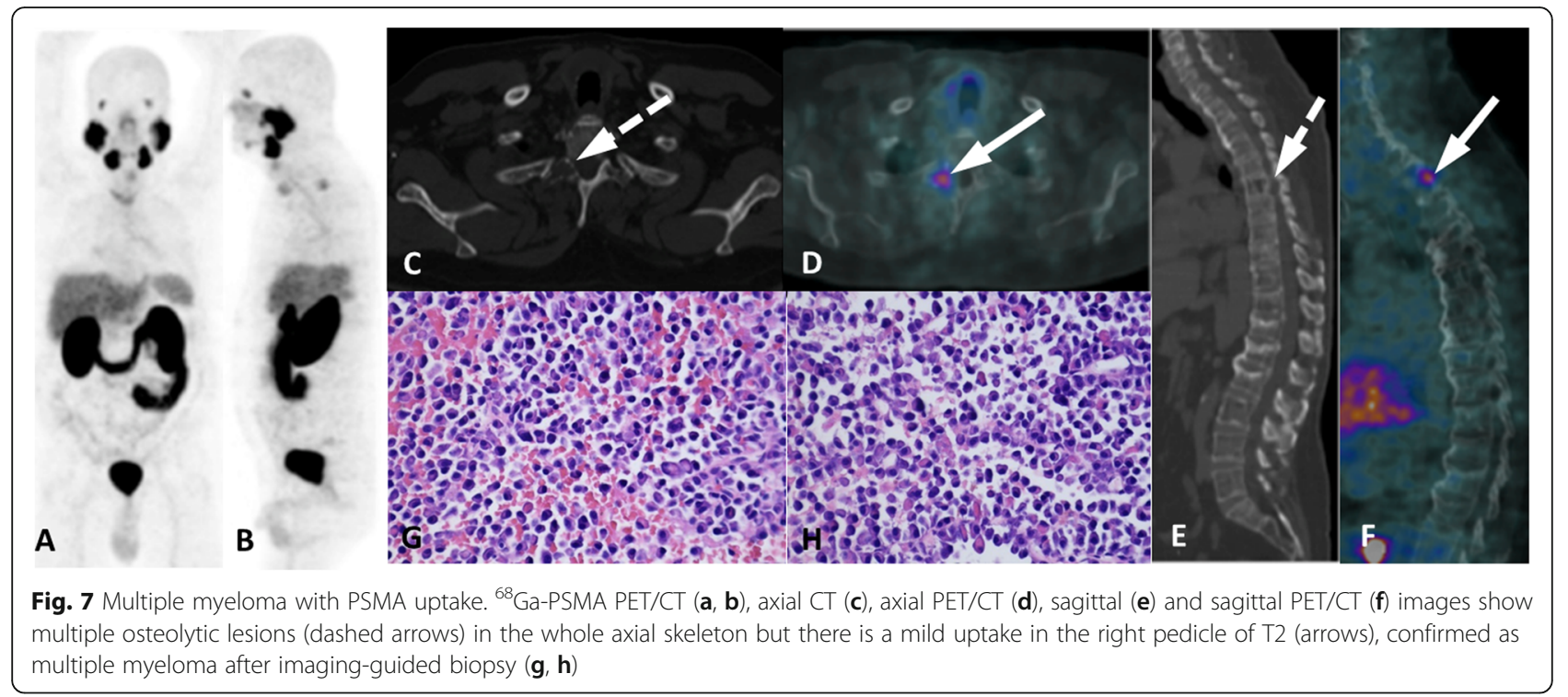

anthracosis [30] and berylliosis [32] have also exhibited PSMA uptake, which reinforces the need for close examination of the patient's clinical history. It is important to highlight that these same conditions do not always present PSMA uptake, even in cases of infectious processes such as histoplasmosis (Fig. 11), and the reason might be related to the grade of associated inflammation, which presents a more or less increase in vascular permeability.

$\mathrm{PCa}$ lesions in the abdomen more often involve the liver but may eventually affect the spleen, pancreas, peritoneum, adrenal and the urinary and gastrointestinal tracts [34]. These lesions are frequently multiple and associated with advanced disease stage along with

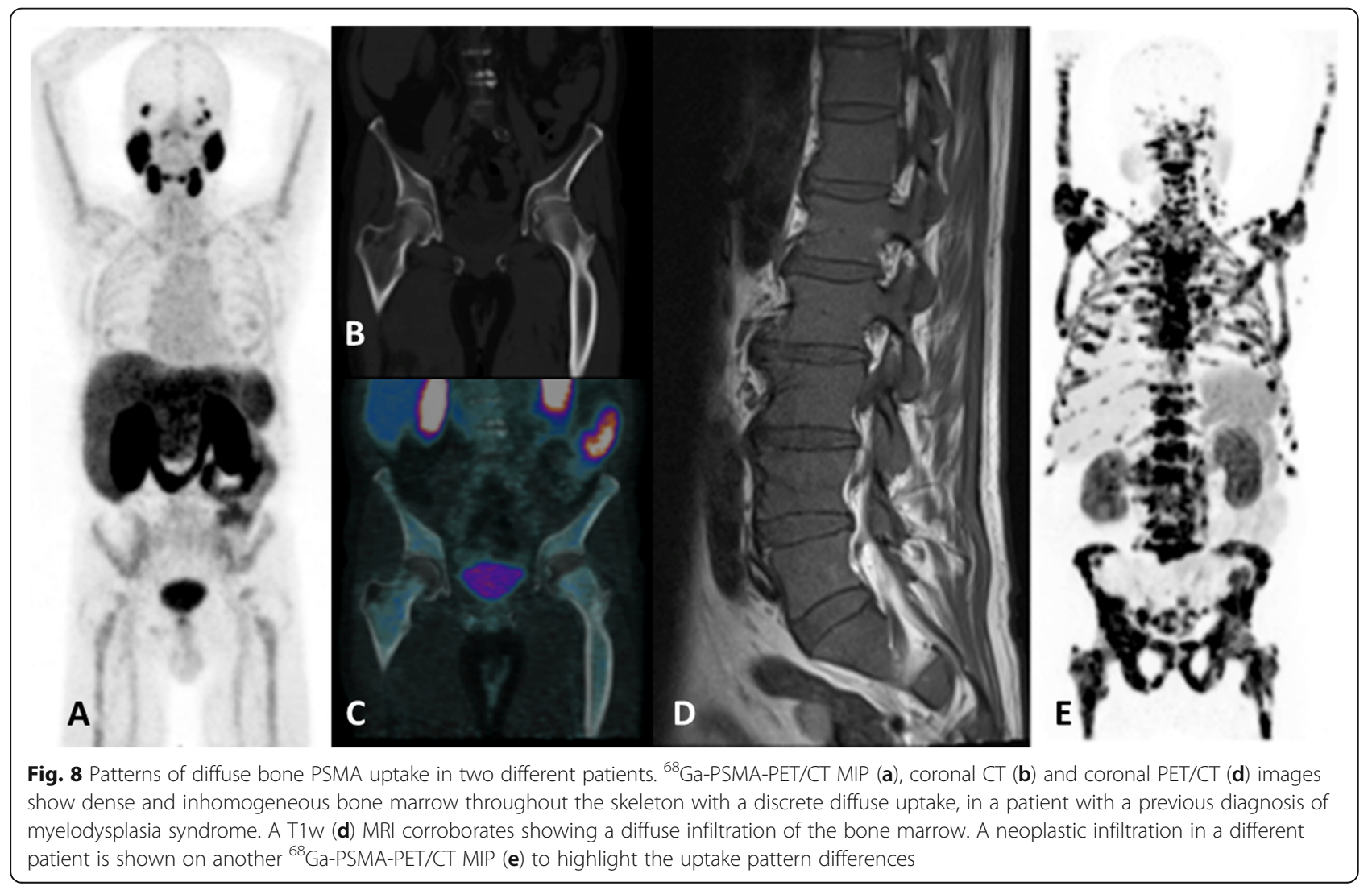




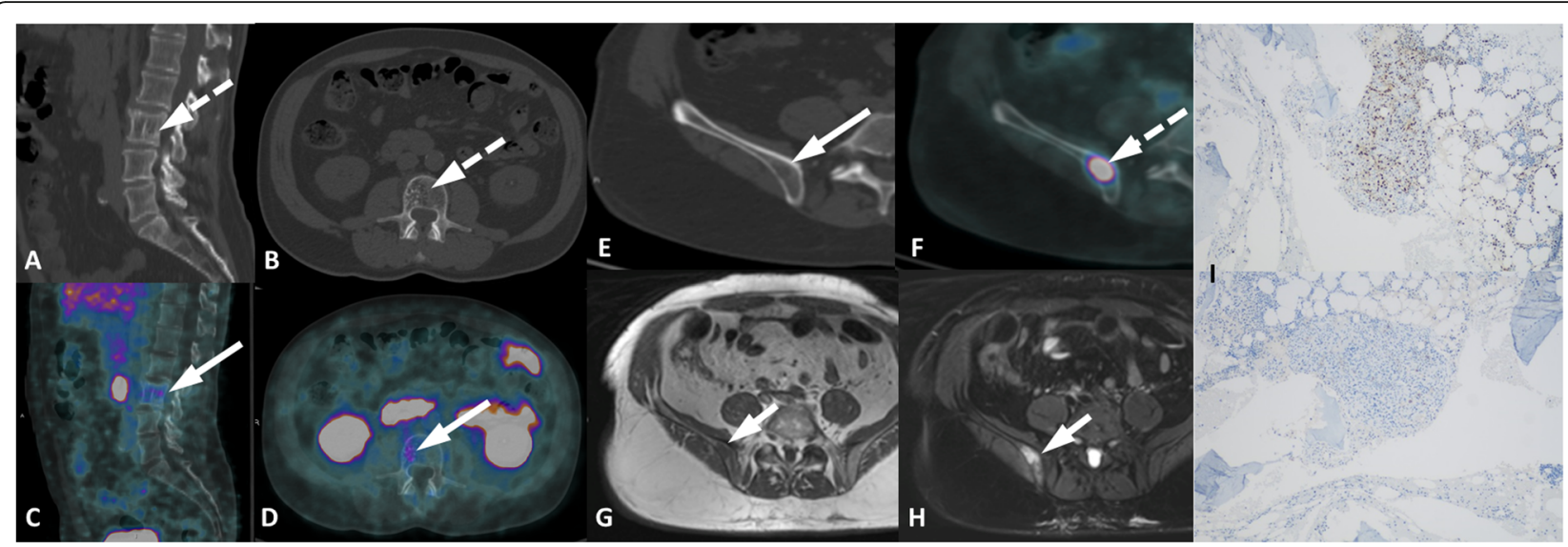

Fig. 9 Different patterns of osseous hemangioma in two patients. Patient 1, spinal hemangioma (a-d). Sagittal CT (a), axial CT (b), sagital PET/CT (c), e axial PET/CT (d) show mild uptake (arrows) in a thickened vertical trabeculae lesion (dashed arrows) surrounded by fat marrow in the right side of L3, typical of hemangioma. Patient 2, iliac hemangioma (e-i). Axial CT (e) and axial PET/CT (f) images showi a lytic lesion (arrow) in the right iliac bone with intense uptake (dashed arrow) and axial T1w (g) and T2W Fat Sat (h) MR images demonstrated a bone lesion without aggressiveness (short arrows). Bone hemangioma was confirmed with percutaneous biopsy and immunohistochemistry (i) showed PSMA expression on the endothelial cells membrane

retroperitoneal nodes and osseous metastases. On the other hand, infectious diseases of the abdomen are mostly restricted to one organ and exhibit substantial morphological changes, such as surrounding fat stranding and prominent reactive lymph nodes. Additional MRI may also be requested for further assessment of morphology, especially in cases involving the pelvis (Fig. 12), cases in which multiple treatments were performed, such as surgery and radiotherapy, and cases in which the CT, even with intravenous contrast, is limited to defining the proper anatomy. Sometimes, however, differentiation to a secondary malignancy is difficult, and a biopsy is mandatory for differential diagnosis (Fig. 13).

In the post-operative setting, prostate bed soft tissue uptake can be seen in the scenario of a very recent radical prostatectomy (< 60 days), when clinicians receive the first PSA test after surgery (6-8 weeks). The expected postprostatectomy PSA level is undetectable, or less than 0.05 or $0.1 \mathrm{ng} / \mathrm{mL}$, and when it is greater than $0.2 \mathrm{ng} / \mathrm{mL}$, it can be considered tumor persistence. In this recent postsurgery status, a common morphological change can be seen as fat stranding around the prostate bed (bladder and urethral anastomosis) and the lateral pelvic wall. Prostate bed fat stranding may exhibit mild and ill-defined PSMA uptake that is slightly higher than that in the background but still seen on maximum intensity projection (MIP) images. This soft tissue uptake can be more intense in early PET acquisition (3 min after tracer injection), while there is a high concentration of radiotracer in the blood. The explanation is not completely known, but it might be related to reparative inflammatory processes and/or increased vascularity. Recognition of this uncommon imaging pattern is important because it can be misinterpreted as a local residual tumor.

In addition to prostatectomy, another pelvic surgical procedure that may present PSMA uptake is inguinal hernia repair. In most inguinal hernia repairs, a mesh prosthesis is placed along the abdominal wall around the deep or superficial inguinal ring, according to surgical technique [36]. Because there is a foreign body associated with such surgery, early or late inflammatory reactions might occur and are not always symptomatic [37]. Another complication described is mesh retraction, which can present as a hyperattenuating, pseudonodular pattern displaced from the original location and can again be associated with graft inflammatory reactions. These findings can exhibit PSMA uptake, which is expected to be mild and diffuse along the mesh foreign body. See Fig. 14.

Pelvic surgeries are more likely to lead to disease recurrence pitfalls; other surgeries might be easier to differentiate from prostate metastasis, but in all scenarios, clinical information is fundamental to help such distinction. When there is a lack of correlation between clinical information and morphological and molecular PET/CT imaging, further imaging evaluation is recommended. MRI could be the best option for further assessment because of its higher soft tissue contrast resolution than that of CT.

\section{Benign neoplasms}

Various benign neoplasms present PSMA uptake, and the number of case reports of these conditions is steadily increasing. False-positive tracer uptake has been especially linked to soft tissue lesions, abnormal vascular 


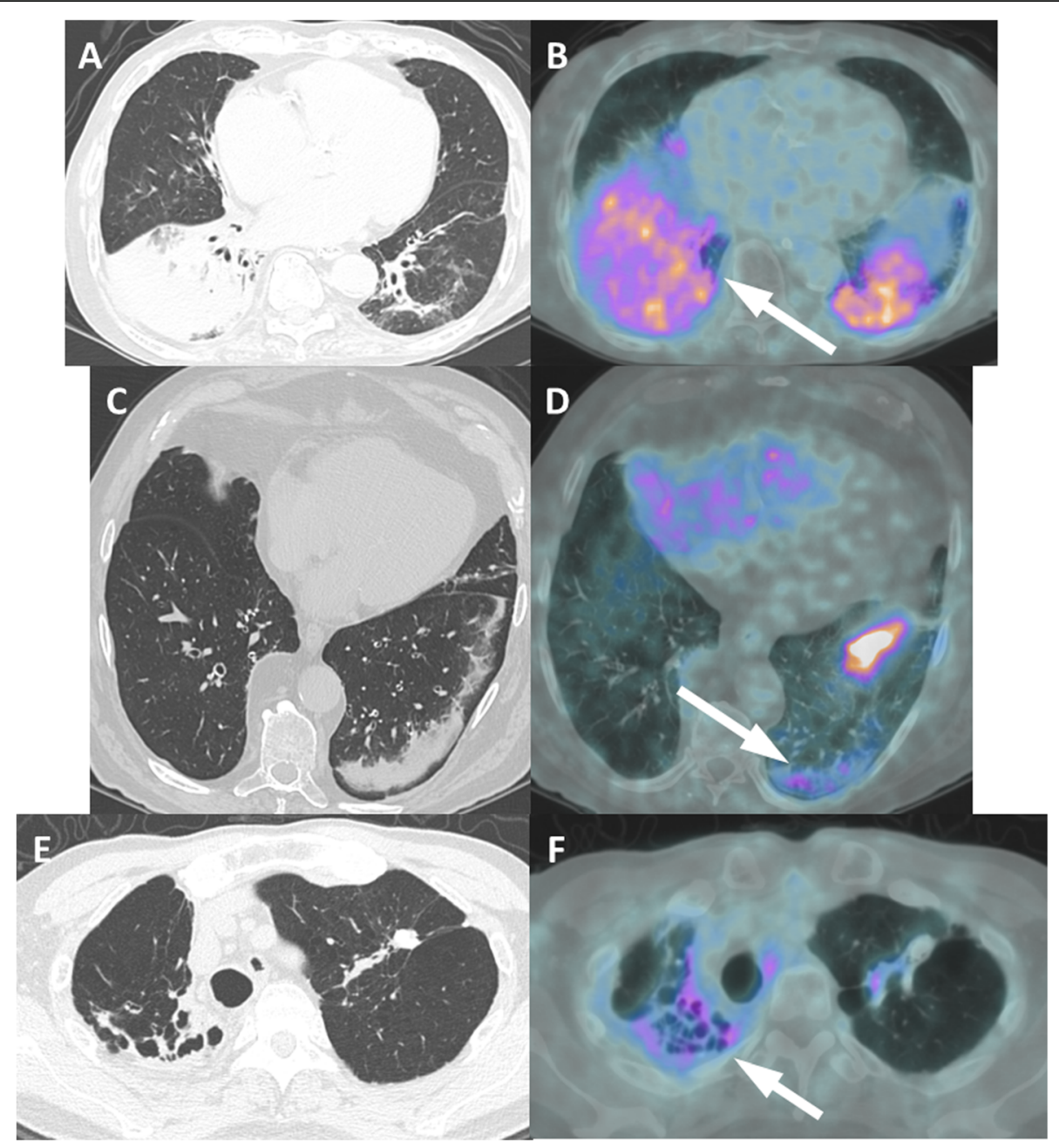

Fig. 10 Pulmonary diseases with PSMA uptake. Patient 1, pulmonary consolidation (a, b). Axial CT (a) and PET/CT (b) show a pulmonary consolidation with air bronchogram in both lower lobes (arrow on B) of the lungs in a 90-year-old patient presenting cough and fever. Patient 2 , pulmonary atelectasis (c, d). Axial CT (c) and PET/CT (d) show a laminar atelectasis in both lower lobes of the lungs with tiny PSMA uptake (arrow on D). Patient 3, tuberculosis sequelae (e, f). Axial CT (e) and PET/CT (f) show retractile bronchiectasis in the upper bilateral lung lobes with a few calcifications and mild uptake (arrow on F), suggestive of tuberculosis sequelae

proliferation, lesions of neurogenic origin, thymoma and adenomas. Most of these lesions could be inferred as not related to PCa due to their location, degree of uptake and clinical and morphological features.

PCa metastases do not commonly affect many of the anatomic sites involved in benign neoplasms, as in the cases of soft tissue lesions or lesions of vascular origin. Soft tissue and musculoskeletal (other than bone) involvement of PCa is unlikely and metastases usually arise in patients with multiple other secondary lesions and high tumor burden. Elastofibroma dorsi, dermatofibroma, acrochordon, fibromatosis desmoid tumor, intramuscular myxoma and pseudoangiomatous stromal hyperplasia of the breast $[4,38]$ are the main entities reported thus far known to present tracer uptake. The lesions may vary from nodules to large soft tissue masses. Common locations include subcutaneous, muscular, tendon, fascial, palmar, plantar, intra-abdominal, abdominal wall, thoracic wall and the breasts $[39,40]$.

The same rationale can be extrapolated to thymomas and thyroid, parathyroid and adrenal adenomas. Thymomas have typical location - mostly well defined, solid, typically rounded, smooth tumors, with a bosselated surface in the anterior mediastinum [41-43].. Thyroid and parathyroid adenomas are readily recognized as thyroid nodules or focal areas of uptake adjacent to the thyroid gland [44]. See Fig. 15. Adrenal adenomas may appear as enlarged adrenals (uni- or bilateral), as a low-density adrenal nodule with attenuation $<10 \mathrm{HU}$ or as an adrenal mass (11). Those entities seldom present challenges to an aware reading physician due to their high prevalence, typical tomographic features and uncommon location for PCa metastases. 


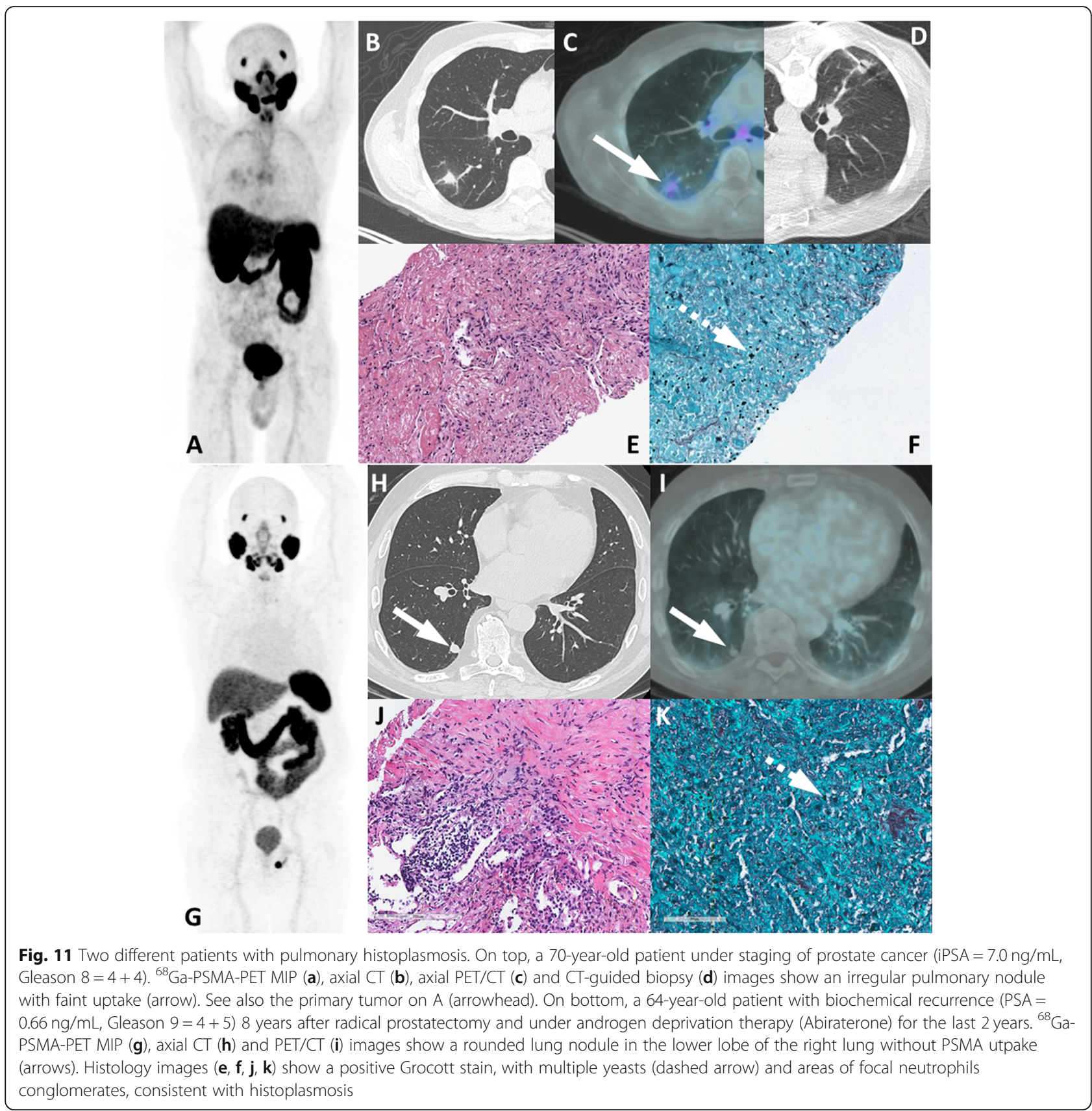

Regarding the level of PSMA expression, most of benign lesions present lower avidity than secondary lesions do, although in a few cases some lesions may present unexpectedly high expression of PSMA. Exceptions to the rule might be found in lesions of vascular origin, such as hemangiopericytoma [45, 46], angiolipoma [47] and hemangiomas [4, 48, 49]. PET findings may be deceptive and tricky and potentially lead to misdiagnosis. The most common presentation is of nodular lesions, mostly of small dimensions, potentially encountered in multiple locations throughout the body $[47,50]$. Hemangiomas are especially worth highlighting, because interpretation of PSMA PET findings may be challenging since they may present intense tracer uptake [48, 49, 51-53]. Typical imaging features include well-defined lesions with low attenuation and slow centripetal enhancement following intravenous contrast administration. Larger lesions may fill with the contrast agent more slowly and heterogeneously than smaller lesions do. Given the dynamic nature of the enhancement pattern, a triphasic contrast-enhanced CT within the PET/CT study may be requested. Common sites of involvement include the liver and the spleen. In both organs, 


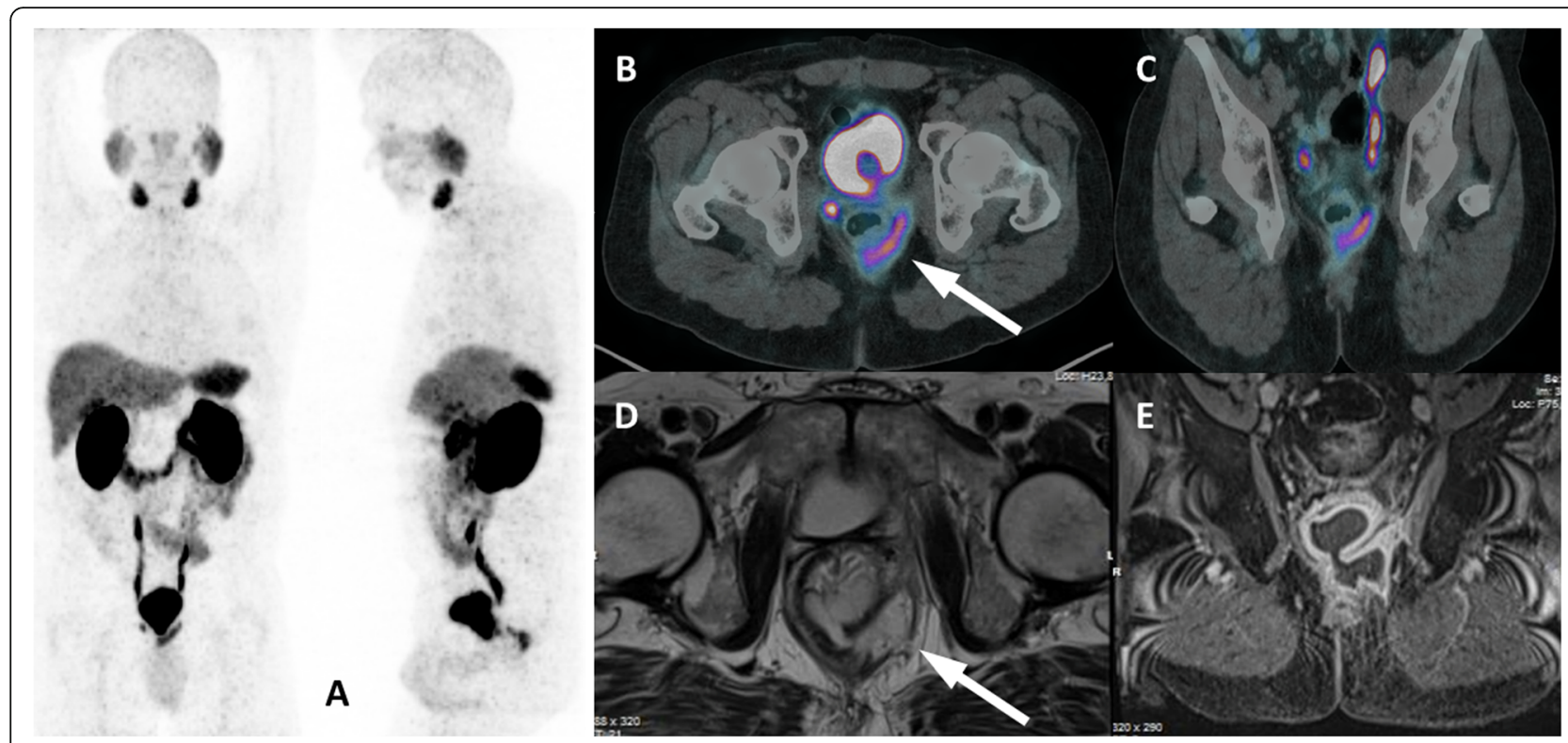

Fig. 12 Perianal fistula with PSMA uptake. ${ }^{68} \mathrm{Ga}$-PSMA-PET MIP (a), axial PET/CT (b), coronal PET/CT (c) and axial T2W MR (d) and coronal T1w with gadolinum (e) show a perirectal fistula in the posterior left ischiorectal fossa (arrows)

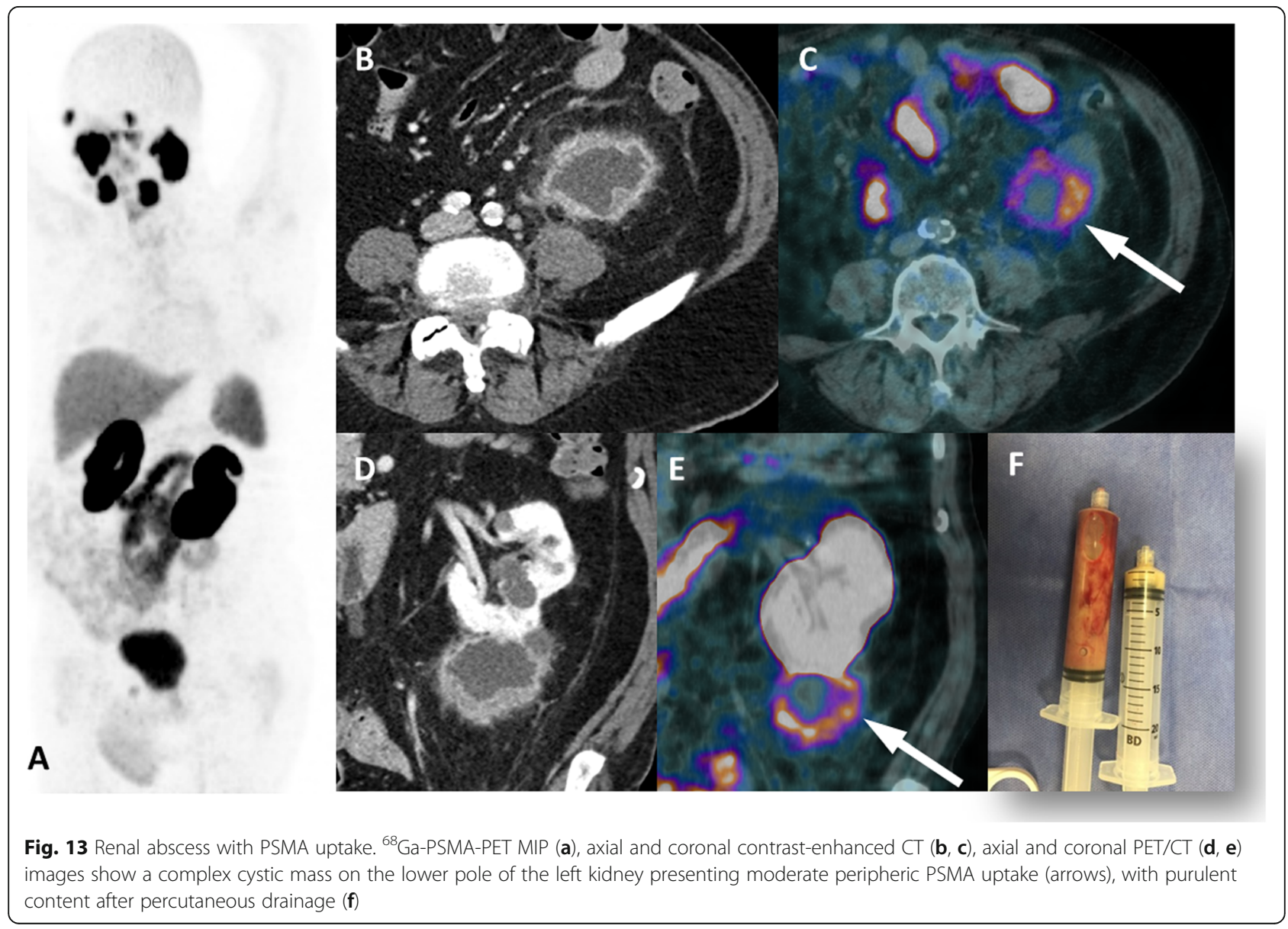




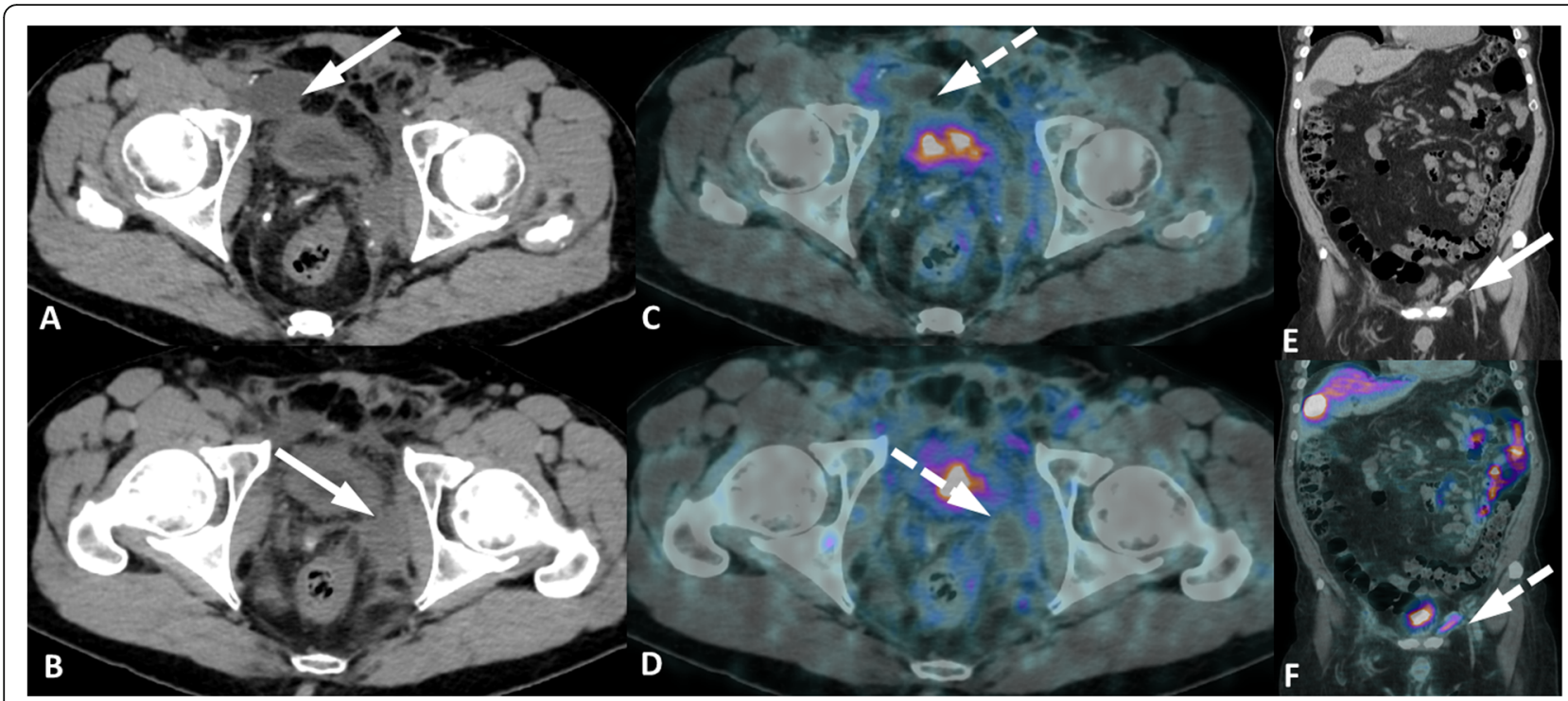

Fig. 14 Post-operative inflammatory findings in two patients. Patient 1, prostatic bed inflammation immediate after radical prostatectomy (a-d). Axial CT (a, b) axial PET/CT (c, d) show faint uptake around bilateral lymphoceles (arrows) and inflammatory changes at bilateral pelvic walls (dashed arrows). Patient 2, inflammatory foreign body reaction of mesh material from left inguinal hernia surgery (e, f). Coronal CT (e) and PET/CT (f) images show an elongated hyperattenuating material along the left inguinal canal (arrow) with mild uptake (dashed arrow)

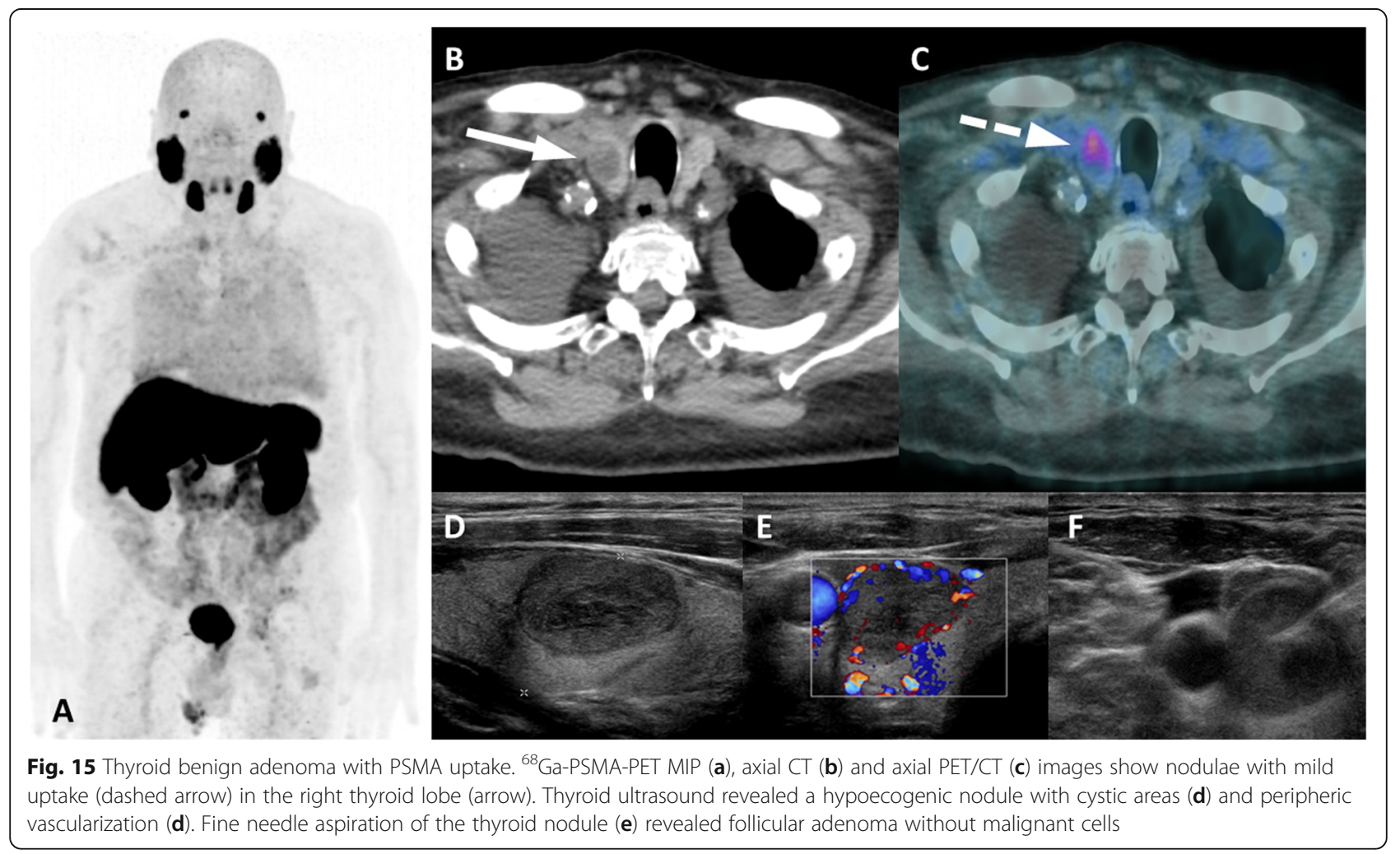



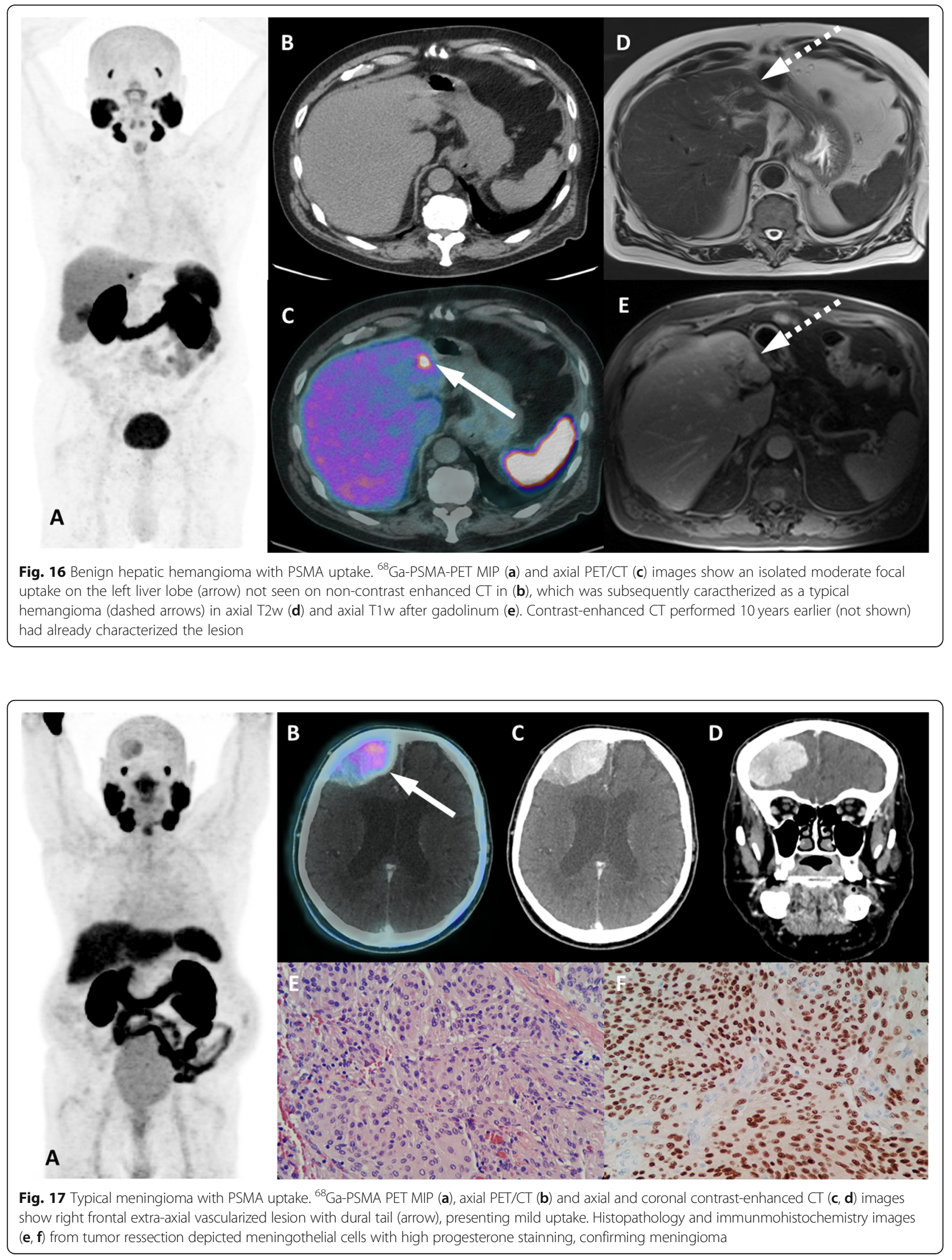


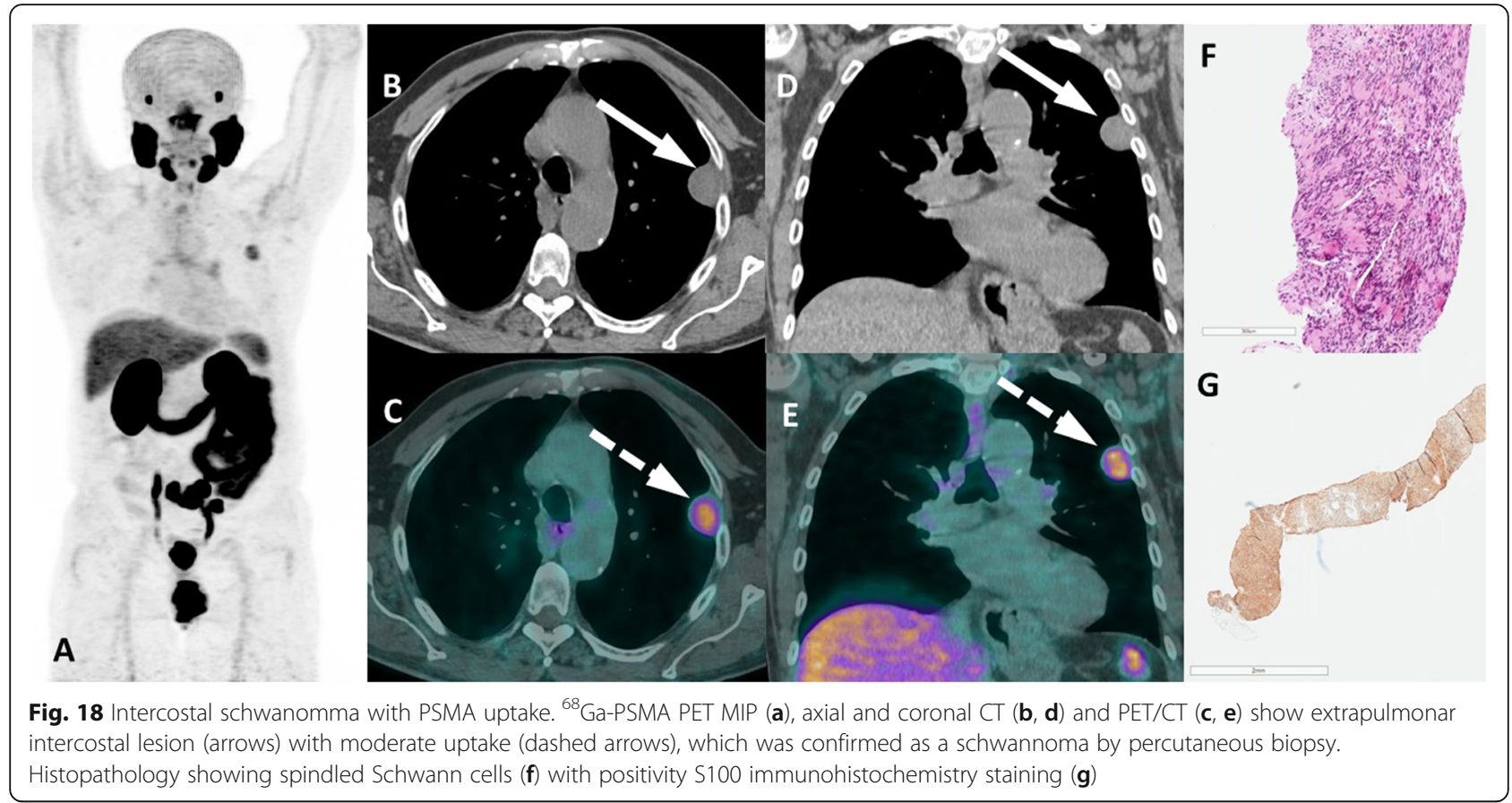

hemangiomas rank among the most prevalent benign lesions, so that lesions presenting PSMA uptake in these organs should have its nature questioned. When findings are equivocal, subsequent evaluation by contrast-enhanced MRI studies may be suggested to aid in clarification (Fig. 16).
A group of entities with variable location and degree of uptake are lesions of neurogenic origin. This category includes meningiomas, schwannomas, peripheral nerve sheath tumors and neurofibromas. Meningiomas may appear in any structure lined by meninges adhered to the internal dural layer. Most lesions are well defined,

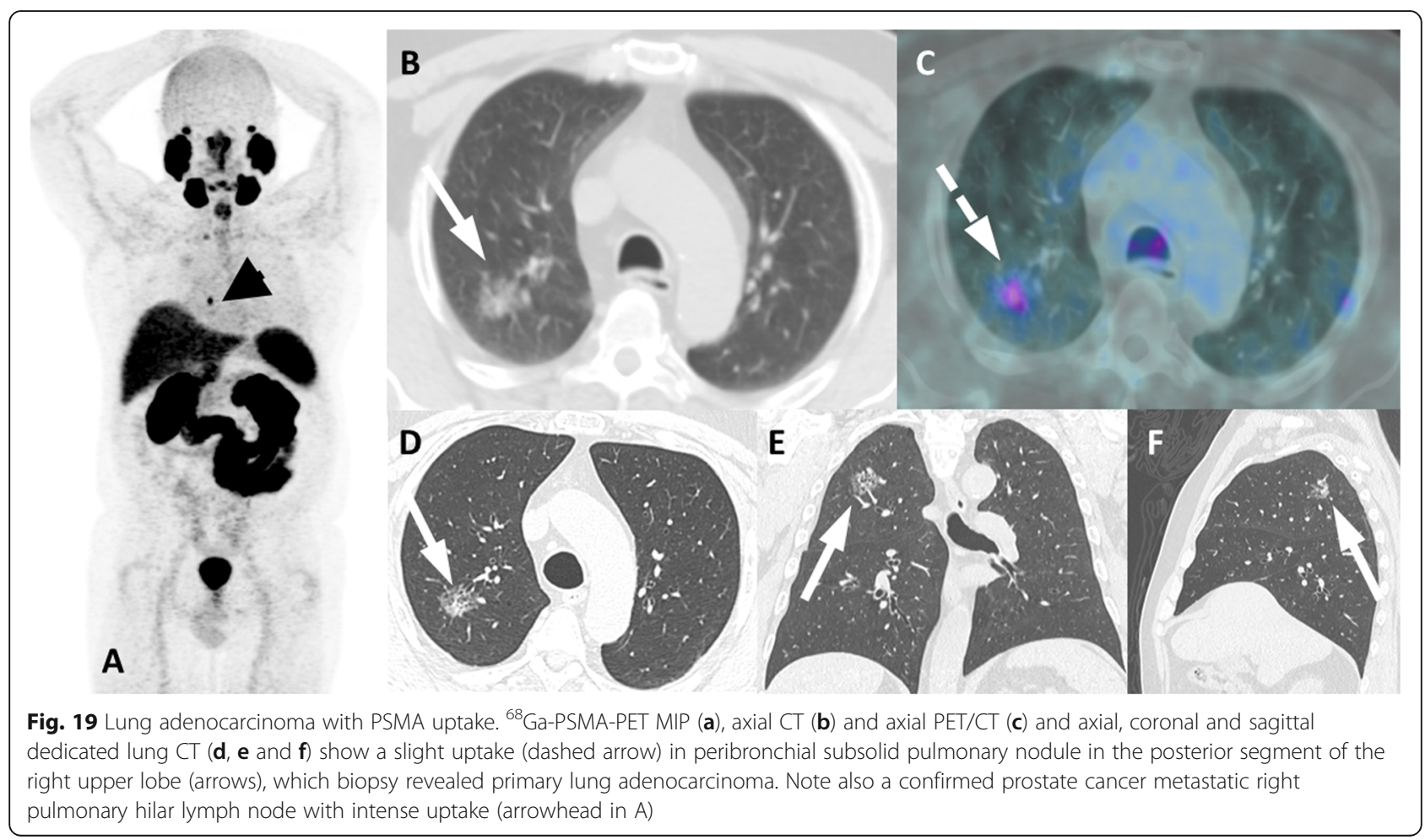




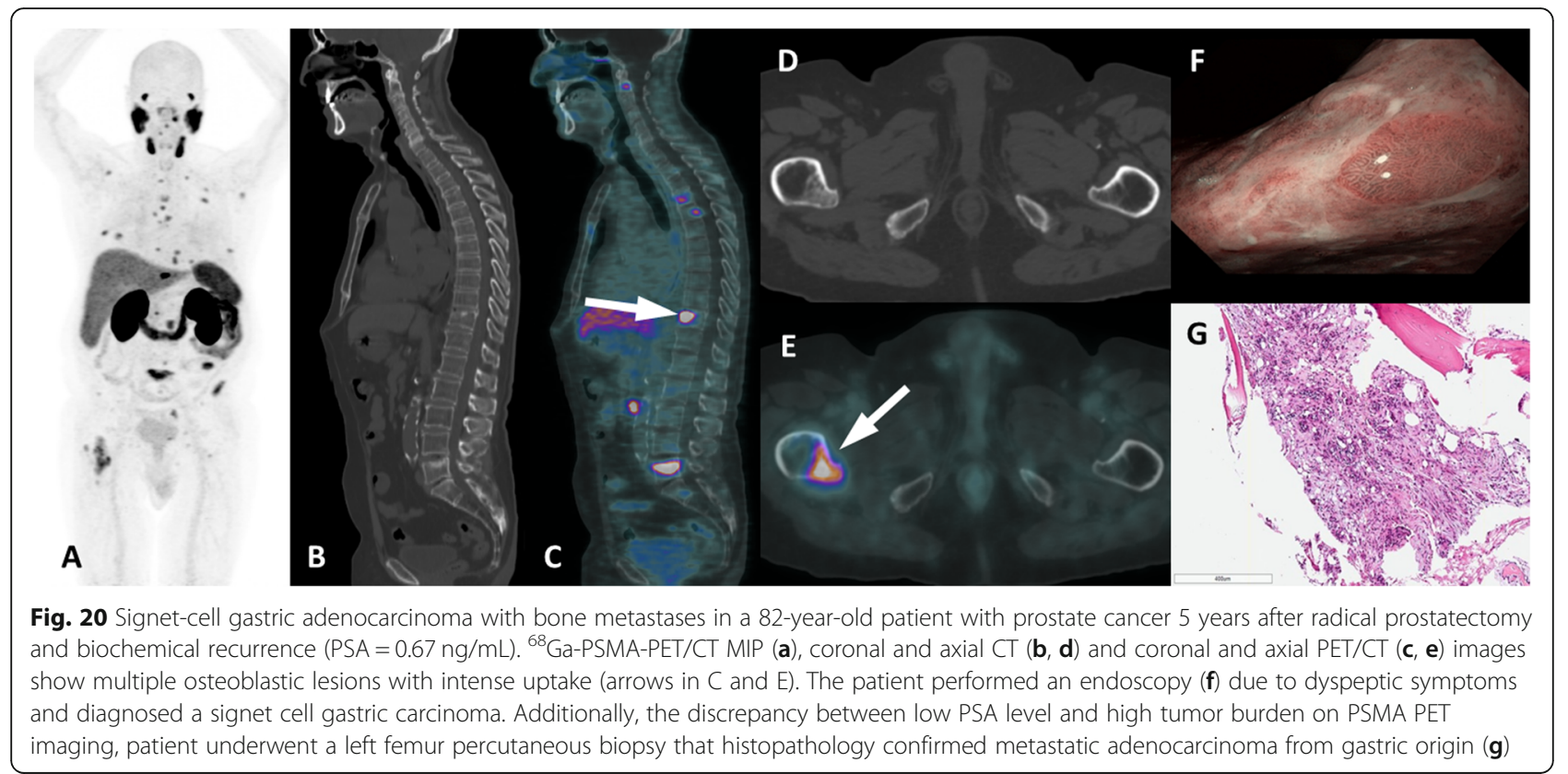

peripherally located, dural based, slightly hyperdense or isodense to normal brain tissue, and may have calcification. There is usually bright and homogenous enhancement of the lesion following the administration of contrast agent. Large lesions might promote adjacent edema or neighbor important brain structures leading to the onset of neurologic symptoms (Fig. 17). In turn, schwannomas may affect intracranial structures, the spine and spinal nerve roots, the trunk (intercostal nerves, the mediastinum, the retroperitoneum, and the gastrointestinal tract) and the limbs (especially flexor surfaces, such as ulnar and peroneal nerves) (Fig. 18).

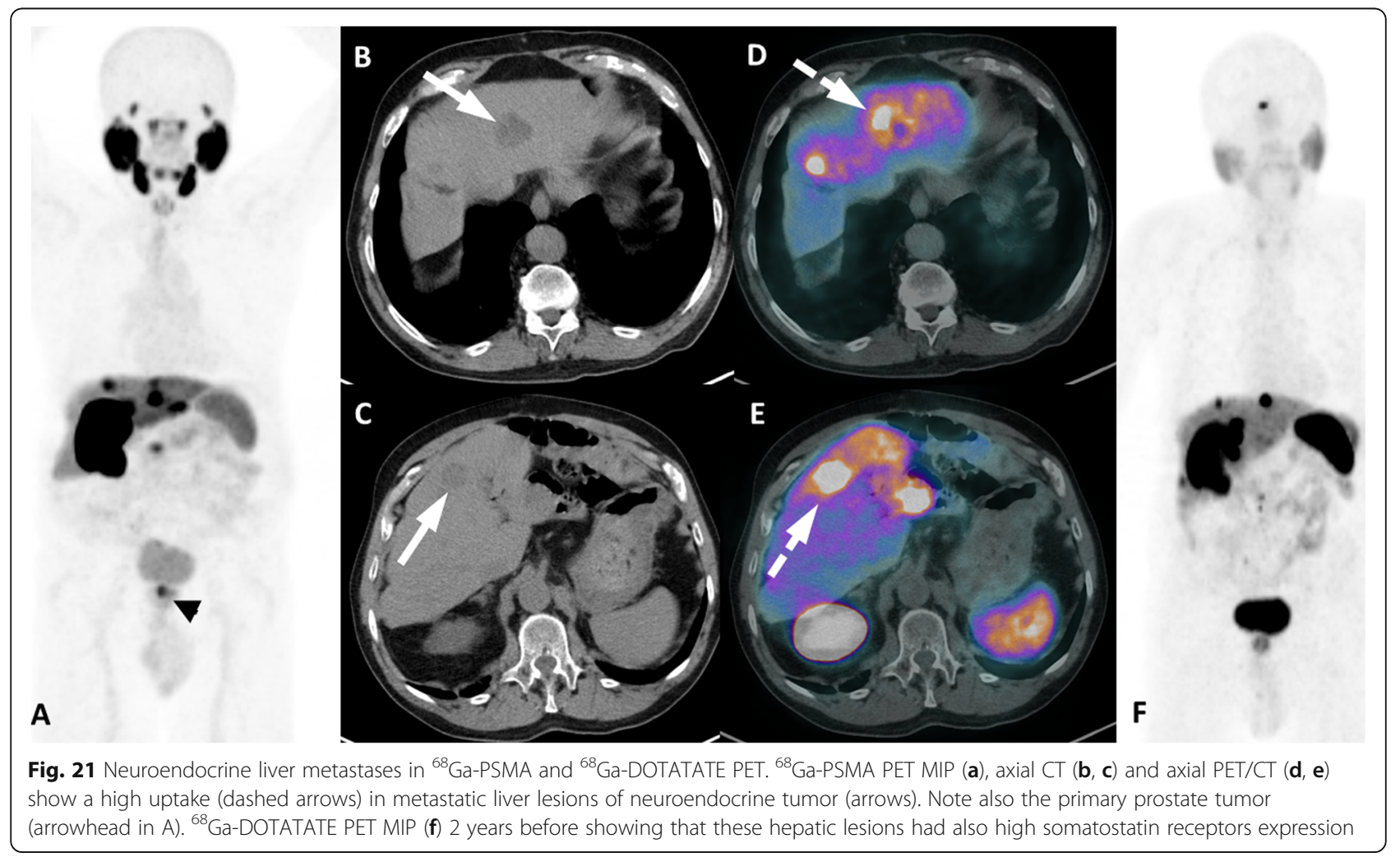




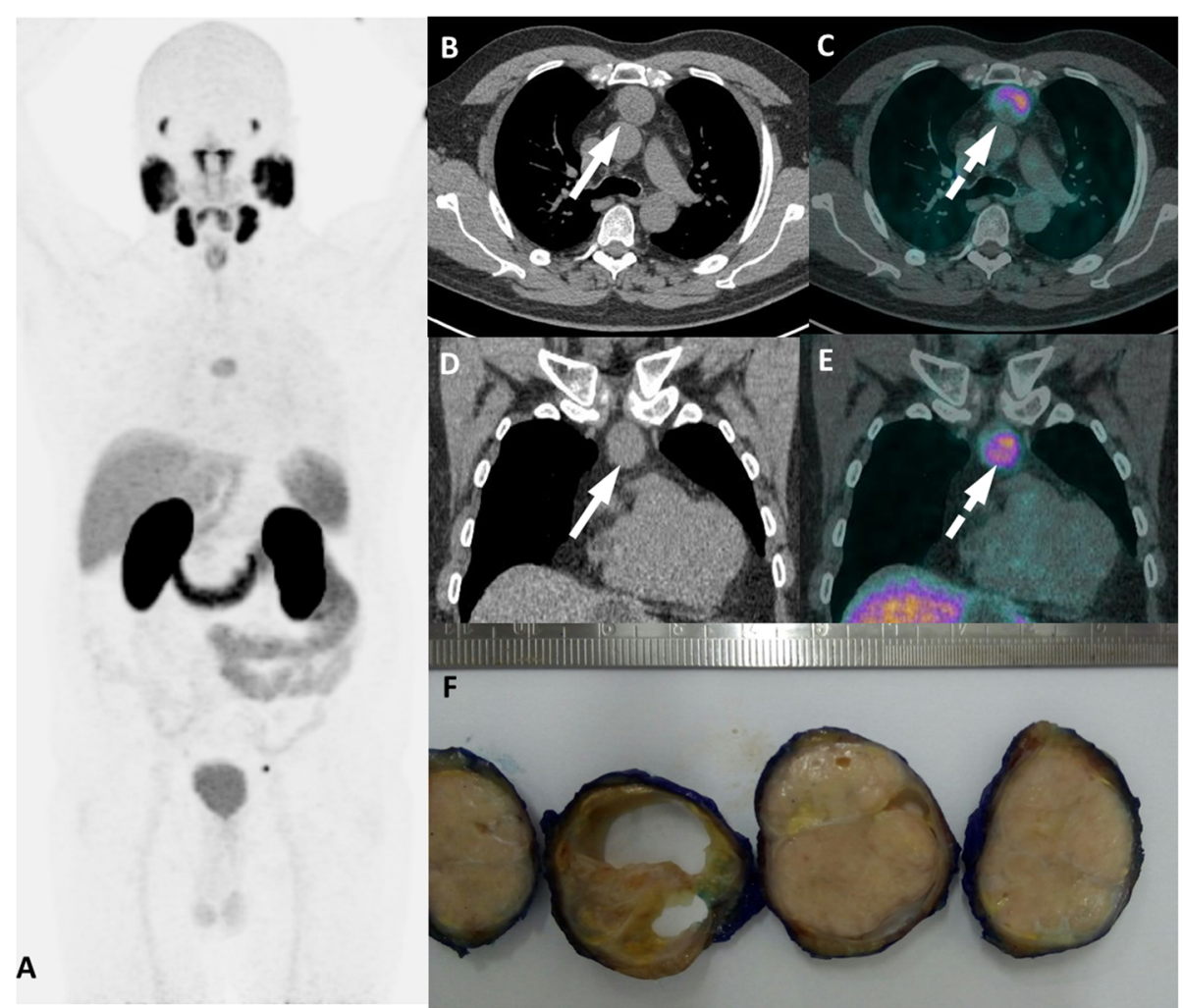

Fig. 22 Thymic carcinoma with PSMA uptake. ${ }^{68} \mathrm{Ga}-\mathrm{PSMA}-\mathrm{PET} / \mathrm{CT}$ MIP (a), axial and coronal CT (b, d) and axial and coronal PET/CT (c, e) images show an anterior mediastinal mass (arrows) with mild uptake (dashed arrows). As an atypical spread for prostate cancer, this lesion was first biopsied and then resected diagnosed as thymoma as shown in pathology macroscopy (f). Case courtesy of Bernardo Bacelar, MD and Thiago F. Nunes, MD

On CT, schwannomas usually appear as well circumscribed nodules or masses of low to intermediate attenuation. Commonly, they dislocate neighboring structures, such as bone, with no signs of invasion, frequently with smooth, corticated edges due to remodeling. They characteristically grow in close proximity to nerves, often along the neuroforamen [54-56]. Finally, neurofibromas are benign peripheral nerve sheath tumors strongly associated with neurofibromatosis type 1 . They appear as well-defined hypodense nodules mostly in subcutaneous tissues, although they may affect almost the entire body. Clinical and morphological features are meaningful for the differential diagnosis of lesions of neurogenic origin, and correlation with MRI may be elucidative in doubtful cases or simply for confirmation purposes.

\section{Malignant neoplasms}

Knowledge of PCa behavior, including typical and atypical disease appearances, was deeply influenced by the emergence of PSMA PET imaging, leading to previously non-imagined imaging features of $\mathrm{PCa}$ [34]. Additionally, with the wide adoption of PSMA PET for PCa, more patients are prone to harboring concomitant malignant neoplasms (by age, genetic or even access selection) and are undergoing highly detailed whole-body examinations, such as PET/CT or PET/MRI, leading to an expected increase in the detection of concomitant nonprostatic malignancies in these patients. As expected, the body of literature available on this topic is rapidly growing, based mainly on case reports of false-positive findings [4, 57-61]. Synchronic malignant neoplasia has been described as rarer and showed lower PSMA uptake than did atypical PCa metastasis and benign lesions in a large cohort of $\mathrm{PCa}$ patients who underwent PSMA PET/CT imaging [62]. However, another large retrospective series with 1889 patients specifically compared lung lesions detected on PSMA PET/CT imaging with presumptive or histological diagnosis, showing that the degree of uptake measured by SUVmax could not differentiate between PCa metastasis and lung cancer [31].

PSMA expression by nonprostatic tumors has been shown by previous immunohistochemistry studies [14, 63-65]. In this context, renal cell carcinoma (RCC) is one of the first and most assessed tumors by PSMA $\mathrm{PET} / \mathrm{CT}$ imaging in the literature because it is a heavily vascularized tumor with well-documented PSMA expression in the neovasculature, as shown by immunohistochemistry and imaging studies $[64,66]$. However, the 


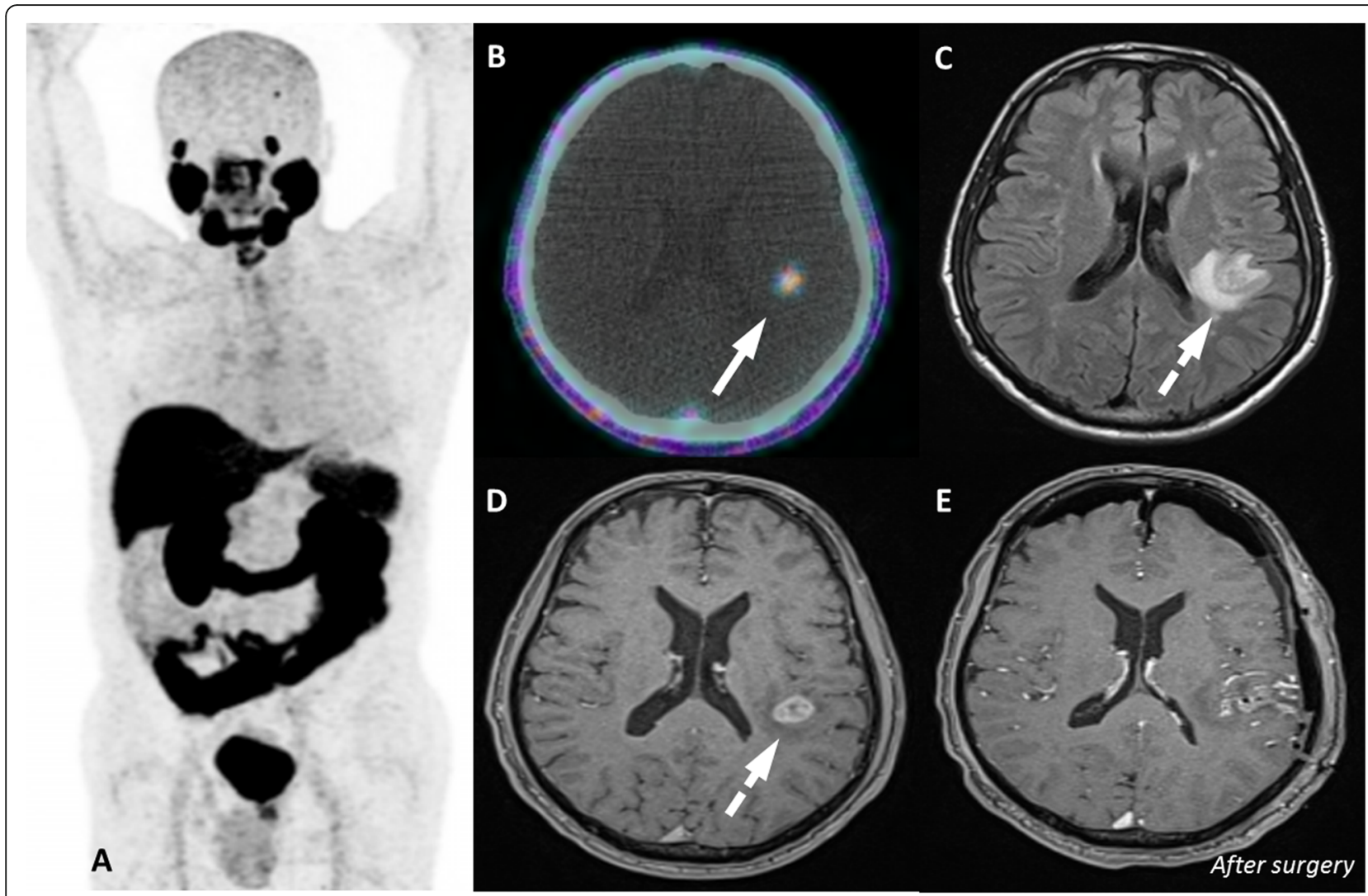

Fig. 23 Glioblastoma multiforme with PSMA uptake. ${ }^{68} \mathrm{Ga}$-PSMA PET MIP (a), axial PET/CT (b), axial FLAIR (c) and T1w after gadolinium (d) images show a mild uptake in a cortico-subcortical lesion on the left parietal lobe (arrows) corresponding to a necrotic lesion with anelar enhancement and adjacent vasogenic edema (dashed arrows), confirmed as a Glioblastoma after surgical resection (e)

emergence and rapid worldwide adoption of PSMA PET imaging for PCa has produced many anecdotal reports of unintentional and potential confounding or falsepositive PSMA ligand uptake in several cancers, such as lung, colorectal, gastric, pancreatic, and thyroid cancers, as well as sarcomas, lymphomas and other tumors [2].

In concordance with the authors' experience and as naturally expected, more prevalent neoplasms in men are responsible for the most common findings of nonprostate malignancies in PSMA PET imaging. Lung cancer can produce challenging findings and has been the subject of some studies. The CT presentation of lesions is an important (not rarely enlightening) step in analysis, and features such as the number, the size, the presence of spiculated (an important marker of suspicion for primary malignancy) or smooth borders and the presence of ground-glass components can be valuable for interpretation (Fig. 19). In a scenario of nonmetastatic or non-advanced metastatic PCa, a lung lesion that may alter patient management should be clarified by biopsy, regardless of the degree of PSMA ligand uptake. This approach can also be helpful for nonpulmonary equivocal lesions.
Central nervous system (CNS) primary tumors (gliomas) may also overexpress PSMA [2]. Moreover, there is a significant difference in PSMA expression between low and high-grade gliomas, explained by the difference in their neovasculature [67]. Isolated CNS PCa metastases are extremely uncommon, so correlation with current disease staging while facing a brain lesion on PSMA is mandatory to consider further investigation and exclude a primary tumor that are often more aggressive than PCa.

Colorectal cancer is another condition in which PSMA expression has been reported [59, 60]. Specifically, for pelvic lesions, in our experience, the degree of uptake and CT pattern showing wall thickening or colon-based lesions can be helpful in distinguishing equivocal lesions from prostate atypical metastases or direct rectal invasion by locally advanced PCa [34]. A particularly confounding site is the liver, a frequent site of colorectal metastasis, where PCa metastasis can exhibit reduced expression of PSMA, reducing the specificity. A valuable clue is that PCa metastasis to the liver is infrequent in nonadvanced stages of disease; therefore, hepatic lesions in this setting must be submitted to additional diagnostic workups, such as dedicated MRI, 


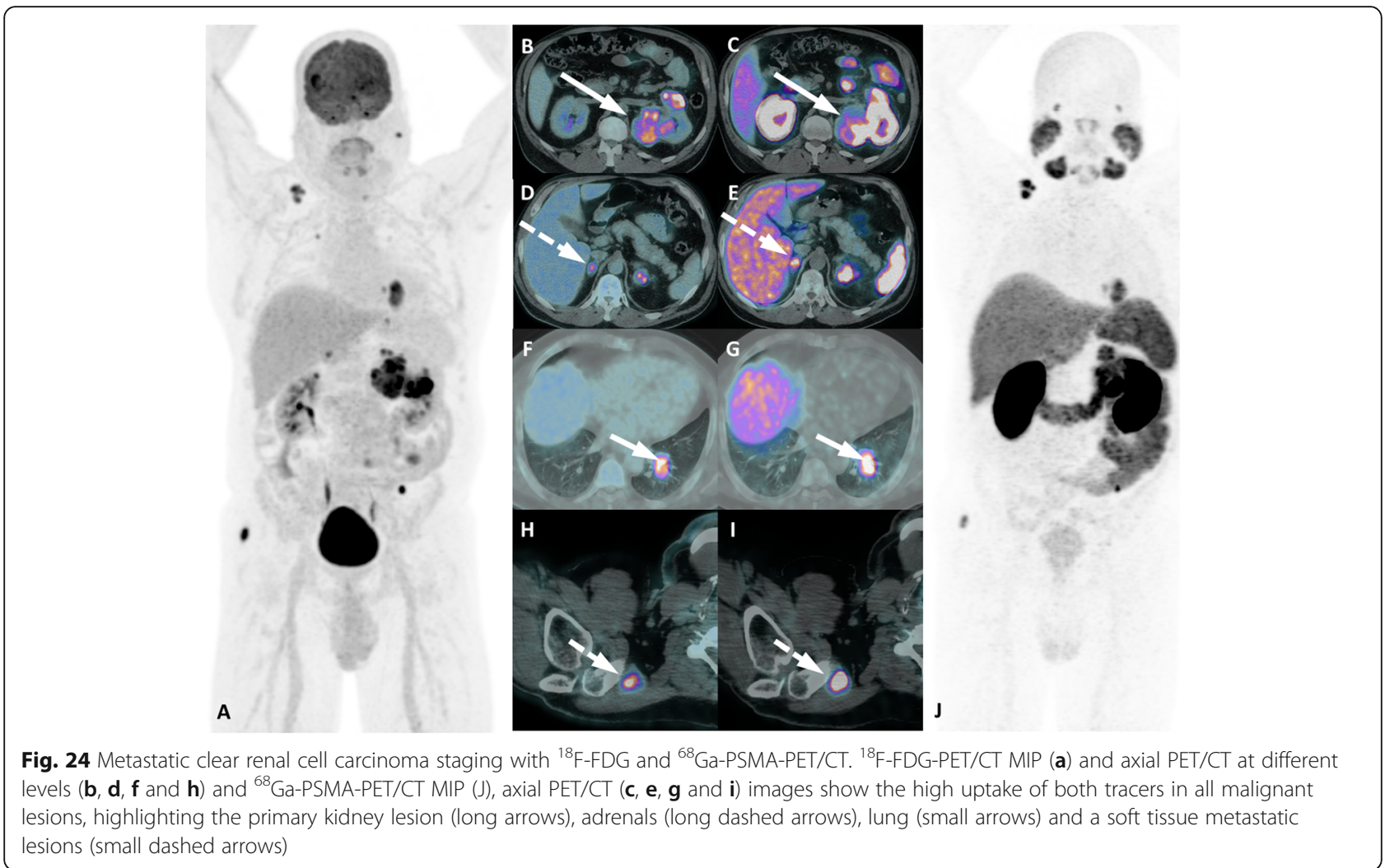

because even primary hepatic neoplasms can also exhibit PSMA expression [68].

Gastric and pancreatic cancers are other sources of potential PSMA-expressing lesions because these tumors also have documented high expression of PSMA in their neovasculature [69] (Fig. 20). Because these organs are very atypical sites of $\mathrm{PCa}$ metastasis, one should automatically think in synchronicity when finding a gastric or pancreatic lesion. For pancreatic tail lesions, neuroendocrine etiology can be suspected, and in some doubtful

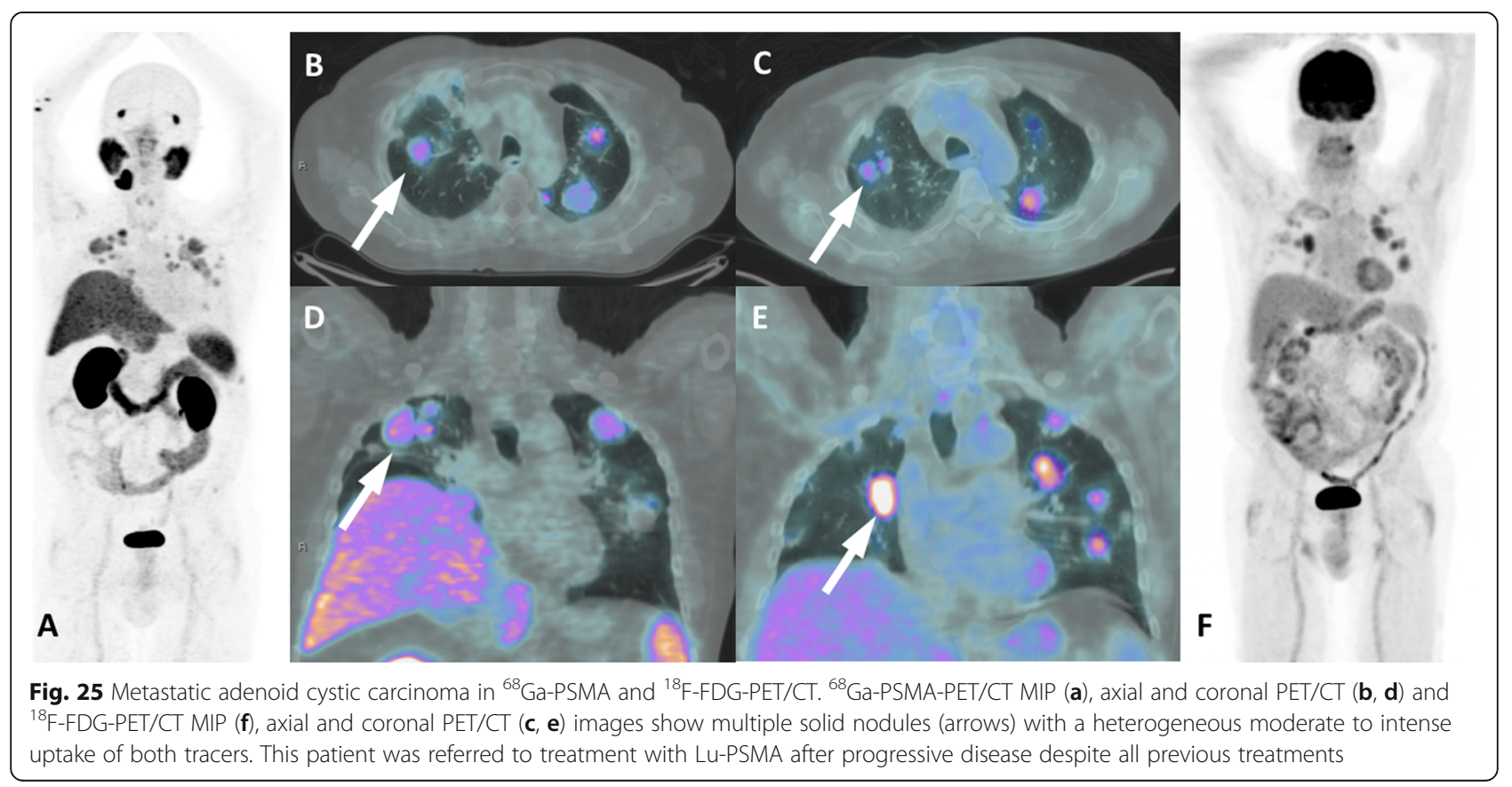


situations, ${ }^{68}$ GA-DOTATATE PET/CT or PET/RM can provide a resolute evaluation (Fig. 21).

Several other malignant neoplasms can exhibit PSMA ligand uptake on PET imaging [4, 70], and the abovementioned principles of interpretation and contextualization of the imaging findings will help reading physicians to suspect nonprostatic lesions or to recommend further appropriate investigations to better assess lesion etiology (Figs. 22 and 23).

\section{Perspectives - from immunobiological knowledge and imaging pitfalls to potential clinical usage}

As a new imaging modality, PSMA PET has shown emerging pitfalls in the medical literature. Such pitfalls may serve as an initial disadvantage that can be turned in an opportunity, since new conditions (especially nonprostatic tumors) can be imaged and perhaps in the future might even be considered for PSMA ligand radiopharmaceuticals in a theranostic approach, which is increasingly being used for PCa [71]. Additionally, previous immunobiological knowledge about PSMA expression in nonprostatic disease (particularly in malignant neoplasms) can now be applied and magnified by an in vivo evaluation in a highly sensitive, whole-body imaging modality. Thus, some promising studies have addressed the diagnostic (and therapeutic) potential of PSMA ligand radiopharmaceuticals in non-PCa malignant neoplasms. RCC is the most studied neoplasm within this new context of an "intentional" use of PSMA ligands, since RCC was one of the first recognized neoplasms with high PSMA expression in endothelial tumor neovasculature (Fig. 24). A pilot study prospectively compared the PSMA PET and CT findings of 10 patients (36 lesions) with metastatic RCC, showing not only higher sensitivity of PSMA PET (92 vs 69\%) but also changes in intended management [72].

Salivary gland tumors are another good example in which a physiological characteristic of PSMA ligand uptake was explored to develop a potential clinical use of the technique. A preliminary study of advanced salivary adenoid cystic carcinomas showed promising results in 10 patients, including high concordance with $\left[{ }^{18} \mathrm{~F}\right]$ fluorodeoxyglucose (FDG)-PET/CT findings and immunoexpression of PSMA on tumor cells themselves (luminal staining showed a highly overregulated profile of PSMA expression that is rarely seen in non-PCa tumors) [73]. Linked to this, a clinical trial (NCT 03319641 - clinicaltrials.gov) tried to form a possible rationale for therapeutic applications in adenoid cystic carcinoma by evaluating the uptake of ${ }^{68} \mathrm{Ga}$-PSMA on PET/CT scans. In our experience, PSMA PET/CT has shown good performance, even in comparison with ${ }^{18} \mathrm{FDG}$ PET/CT when evaluating adenoid cystic carcinoma of the salivary glands (Fig. 25).
Following the same rationale, diseases in which other modalities (including ${ }^{18} \mathrm{FDG}$-PET/CT) have left some unmet needs are being studied for potential indications for PSMA-ligand PET imaging (and even with theranostic intention). Examples include breast cancer (especially the lobular subtype), thyroid cancer, pancreatic cancer, brain tumors and others $[65,74]$.

\section{Conclusions}

Nonprostatic diseases exhibiting PSMA uptake on PET are becoming more common as the number of performed scans increases. The first step to differentiate them from metastatic $\mathrm{PCa}$ is recognizing the variety of conditions presenting PSMA uptake on PET imaging, from inflammatory processes and benign tumors to bone lesions and malignant neoplasms. Understanding the biology behind PSMA uptake represents another primer that favors this distinction. PSMA expression in the apical membrane, which is a marker of PCa cells, presents greater tracer uptake on PET imaging than the level of expression shown in the cytoplasm, which is more often observed in nonprostatic diseases. However, this finding does not enable a clear differentiation between PCa lesions and nonprostatic diseases. Several other characteristics should be considered, such as the pattern of disease spread, the number of lesions and mandatory examinations of patient history and PSA levels. The correlation of PSMA PET to CT and/or MRI morphology, however, is a more assertive analysis tool, since some conditions might present specific anatomical features. Despite the initial thought of PSMA expression in nonprostatic diseases (usually related to endothelial expression in associated neovasculature) as a drawback, these unintentional findings have paved the way for the application of PSMA PET imaging as an additional diagnostic tool for them.

\section{Abbreviations \\ CT: Computed tomography; FDG: Fluorodeoxyglucose; MRI: Magnetic resonance imaging; PCa: Prostate cancer; PET: Positron-emission tomography} PSMA: Prostate-specific membrane antigen

\section{Acknowledgements \\ Not applicable.}

\section{Authors' contributions}

FGB, MAQ, RFN and JFGM made substantial contributions to the conception and design of the work. FGB, MAQ, RFN, LBC, ECZ and JFGM have drafted the work. GGC and CAB contributed to the conception of the work and substantively revised it. All authors agreed to participate and approved the submitted version.

\section{Funding}

There was no funding for this particular review.

Availability of data and materials

Yes

Ethics approval and consent to participate Not applicable. 


\section{Consent for publication}

Yes

\section{Competing interests}

The authors declare that they have no competing interests.

Received: 28 November 2019 Accepted: 2 March 2020

Published online: 14 March 2020

\section{References}

1. Stoykow C, Huber-Schumacher S, Almanasreh N, Jilg C, Ruf J. Strong PSMA Radioligand uptake by rectal carcinoma: who put the "S" in PSMA? Clin Nucl Med. 2017:42:225-6.

2. Malik D, Kumar R, Mittal BR, Singh H, Bhattacharya A, Singh SK. 68Ga-labeled PSMA uptake in nonprostatic malignancies: has the time come to remove "PS" from PSMA? Clin Nucl Med. 2018;43:529-32.

3. Perera M, Papa N, Roberts M, Williams M, Udovicich C, Vela I, et al. Gallium68 prostate-specific membrane antigen positron emission tomography in advanced prostate cancer-updated diagnostic utility, sensitivity, specificity, and distribution of prostate-specific membrane antigen-avid lesions: a systematic review and meta-analysis. Eur Assoc Urol. 2019. https://doi.org/ 10.1016/j.eururo.2019.01.049. [Epub ahead of print].

4. Backhaus P, Noto B, Avramovic N, Grubert LS, Huss S, Bögemann M, et al. Targeting PSMA by radioligands in non-prostate disease-current status and future perspectives. Eur J Nucl Med Mol Imaging. 2018;45:860-77.

5. Demirci E, Sahin OE, Ocak M, Akovali B, Nematyazar J, Kabasakal L. Normal distribution pattern and physiological variants of 68Ga-PSMA-11 PET/CT imaging. Nucl Med Commun. 2016;37:1169-79.

6. Prasad V, Steffen IG, Diederichs G, Makowski MR, Wust P, Brenner W. Biodistribution of [68Ga] PSMA-HBED-CC in patients with prostate cancer: characterization of uptake in Normal organs and tumour lesions. Mol Imaging Biol. 2016;18:428-36.

7. Giesel FL, Hadaschik B, Cardinale J, Radtke J, Vinsensia M, Lehnert W, et al. F18 labelled PSMA-1007: biodistribution, radiation dosimetry and histopathological validation of tumor lesions in prostate cancer patients. Eur J Nucl Med Mol Imaging. 2017;44:678-88.

8. Horoszewicz JS, Kawinski E, Murphy GP. Monoclonal antibodies to a new antigenic marker in epithelial prostatic cells and serum of prostatic cancer patients. Anticancer Res. 1987;7:927-35.

9. Will L, Sonni I, Kopka K, Kratochwil C, Giesel FL, Haberkorn U. Radiolabeled prostate-specific membrane antigen small-molecule inhibitors. Q J Nucl Med Mol Imaging. 2017;61:168-80.

10. Rajasekaran SA, Anilkumar G, Oshima E, Bowie JU, Liu H, Heston W, et al. A novel cytoplasmic tail MXXXL motif mediates the internalization of prostatespecific membrane antigen. Mol Biol Cell. 2003;14:4835-45.

11. Liu H, Rajasekaran AK, Moy P, Xia Y, Kim S, Navarro V, et al. Constitutive and antibody-induced internalization of prostate-specific membrane antigen. Cancer Res. 1998;58:4055-60.

12. O'Keefe DS, Bacich DJ, Huang SS, Heston WDW. A perspective on the evolving story of PSMA biology, PSMA-based imaging, and Endoradiotherapeutic strategies. J Nucl Med. 2018;59:1007-13.

13. Carter RE, Feldman AR, Coyle JT. Prostate-specific membrane antigen is a hydrolase with substrate and pharmacologic characteristics of a neuropeptidase. Proc Natl Acad Sci U S A. 1996;93:749-53.

14. Mhawech-Fauceglia P, Zhang S, Terracciano L, Sauter G, Chadhuri A Herrmann FR, et al. Prostate-specific membrane antigen (PSMA) protein expression in normal and neoplastic tissues and its sensitivity and specificity in prostate adenocarcinoma: an immunohistochemical study using mutiple tumour tissue microarray technique. Histopathology. 2007;50:472-83.

15. Rischpler C, Eiber M, Beck TI, Schlitter AM, Gschwend J, Meyer PT, et al. 68 Ga-PSMA-HBED-CC uptake in cervical, celiac, and sacral ganglia as an important pitfall in prostate cancer PET imaging. J Nucl Med. 2018;59: 1406-11.

16. Chang SS, O'Keefe DS, Bacich DJ, Reuter VE, Heston WD, Gaudin PB. Prostate-specific membrane antigen is produced in tumor-associated neovasculature. Clin Cancer Res. 1999;5:2674-81.

17. Hermann RM, Djannatian M, Czech N, Nitsche M. Prostate-specific membrane antigen PET/CT: false-positive results due to sarcoidosis? Case Rep Oncol. 2016;9:457-63.

18. Jochumsen MR, Bouchelouche K. Intense 68Ga-PSMA uptake in diverticulum of the sigmoid colon. Clin Nucl Med. 2018;43:110.
19. Klingenberg S, Jochumsen MR, Nielsen TF, Bouchelouche K. 68Ga-PSMA uptake in anal fistula on PET/CT scan. Clin Nucl Med. 2019;44:e54.

20. Hofman MS, Hicks RJ, Maurer T, Eiber M. Prostate-specific membrane antigen PET: clinical utility in prostate cancer, Normal patterns, pearls, and pitfalls. RadioGraphics. 2018;38:200-17.

21. Sheikhbahaei $S$, Afshar-Oromieh A, Eiber M, Solnes LB, Javadi MS, Ross AE, et al. Pearls and pitfalls in clinical interpretation of prostate-specific membrane antigen (PSMA)-targeted PET imaging. Eur J Nucl Med Mol Imaging. 2017;44:2117-36.

22. Parihar AS, Vadi SK, Mittal BR, Kumar R, Bal A, Singh SK. 68Ga-PSMA-HBEDCC-avid synchronous urinary bladder Paraganglioma in a patient with metastatic prostate carcinoma. Clin Nucl Med. 2018;43:e329-30.

23. Malik D, Mittal BR, Kumar R, Singh H, Bhattacharya A, Singh SK. Incidental detection of tracer avidity in Liposarcoma on 68Ga-labeled prostate-specific membrane antigen PET/CT. Clin Nucl Med. 2018;43:e334-5.

24. Vaz S, Oliveira C, Castanheira JC, Silva ÂF, Costa DC. Gastric GIST incidentally detected on 68Ga-PSMA-PET/CT: correlation between functional imaging and histology. Clin Nucl Med. 2018;43:e488-91.

25. Jochumsen MR, Dias AH, Bouchelouche K. Benign traumatic rib fracture a potential pitfall on68Ga-prostate-specific membrane antigen PET/CT for prostate cancer. Clin Nucl Med. 2018;43:38-40.

26. Shetty D, Patel D, Le K, Bui C, Mansberg R. Pitfalls in Gallium-68 PSMA PET/ CT interpretation-a pictorial review. Tomography. 2018;4:182-93.

27. Vadi SK, Kumar R, Singh H, Singh SK, Mittal BR. 68Ga-prostate-specific membrane antigen expression in Neurocysticercosis lesions in a patient with prostate carcinoma. Clin Nucl Med. 2018;43:e122-4.

28. Pedersen MA, Bouchelouche K, Vendelbo MH. 68Ga-PSMA uptake in middle lobe syndrome. Clin Nucl Med. 2018;43:e376.

29. Ahuja A, Taneja S, Thorat K, Jena A. 68Ga-prostate-specific membrane antigen-avid tubercular lesions mimicking prostate Cancer metastasis on simultaneous prostate-specific membrane antigen PET/MRI. Clin Nucl Med. 2017:42:e509.

30. McGuiness M, Sounness B. 68Ga-PSMA-ligand PET/CT uptake in Anthracosilicosis. Clin Nucl Med. 2017:42:e431.

31. Pyka T, Weirich G, Einspieler I, Maurer T, Theisen J, Hatzichristodoulou G, et al. 68Ga-PSMA-HBED-CC PET for differential diagnosis of suggestive lung lesions in patients with prostate cancer. J Nucl Med. 2016;57:367-71.

32. Huang $K$, Baur ADJ, Furth C. Prostate-specific membrane antigen-positive manifestations of chronic beryllium lung disease. Clin Nucl Med. 2019;44:64.

33. Janssen J-C, Meißner S, Woythal N, Prasad V, Brenner W, Diederichs G, et al. Comparison of hybrid 68Ga-PSMA-PET/CT and 99mTc-DPD-SPECT/CT for the detection of bone metastases in prostate cancer patients: additional value of morphologic information from low dose CT. Eur Radiol2017/08/06. 2018; 28:610-9.

34. Barbosa FG, Queiroz MA, Nunes RF, Viana PCC, Marin JFG, Cerri GG, et al. Revisiting prostate cancer recurrence with PSMA PET: atlas of typical and atypical patterns of spread. Radiographics. 2019;39:186-212.

35. Janssen JC, Woythal N, Meißner S, Prasad V, Brenner W, Diederichs G, et al. [68Ga] PSMA-HBED-CC uptake in osteolytic, osteoblastic, and bone marrow metastases of prostate cancer patients. Mol imaging biol. Mol Imaging Biol. 2017:19:933-43.

36. Cunningham HB, Weis JJ, Taveras LR, Huerta S. Mesh migration following abdominal hernia repair: a comprehensive review. Hernia. 2019;23(2):235.

37. Delikoukos S, Tzovaras G, Liakou P, Mantzos F, Hatzitheofilou C. Late-onset deep mesh infection after inguinal hernia repair. Hernia. 2007;11:15-7.

38. Gorin MA, Marashdeh W, Ross AE, Allaf ME, Pienta KJ, Pomper MG, et al. Uptake of the prostate-specific membrane antigen-targeted PET radiotracer 18F-DCFPyL in elastofibroma dorsi. Nucl Med Commun. 2017;38:795-8.

39. Murphey MD, Ruble CM, Tyszko SM, Zbojniewicz AM, Potter BK, Miettinen M. Musculoskeletal fibromatoses: radiologic-pathologic correlation. RadioGraphics. 2009:29:2143-83.

40. Lee JC, Thomas JM, Phillips S, Fisher C, Moskovic E. Aggressive fibromatosis: MRI features with pathologic correlation. Am J Roentgenol. 2006;186:247-54.

41. Krishnaraju VS, Basher RK, Singh H, Singh SK, Bal A, Mittal BR. Incidental detection of type B2 Thymoma on 68Ga-labeled prostate-specific membrane antigen PET/CT imaging. Clin Nucl Med. 2018;43:356.

42. Jeong YJ, Lee KS, Kim J, Shim YM, Han J, Kwon OJ. Does CT of Thymic epithelial tumors enable us to differentiate histologic subtypes and predict prognosis? Am J Roentgenol. 2004;183:283-9. 
43. Takahashi K, Al-Janabi NJ. Computed tomography and magnetic resonance imaging of mediastinal tumors. J Magn Reson Imaging. 2010;32:1325-39.

44. Karimov I, Novotny A, Eiber M, Feuerecker B, Weber WA, Jesinghaus M, et al. Pitfalls in Ga-68-PSMA-PET/CT: incidental finding of parathyroid adenoma. Eur J Nucl Med Mol Imaging. 2018;46:81675

45. Lieb WE, Kobe C, Drzezga A, Hilgenberg U, Hammes J. Orbital Hemangiopericytoma in 68Ga-prostate-specific membrane antigen-HBEDCC PET/CT. Clin Nucl Med. 2017;42:812-4.

46. Patro KC, Palla M, Kashyap R. Unusual case of metastatic intracranial hemangiopericytoma and emphasis on role of 68Ga-PSMA PET in imaging. Clin Nucl Med. 2018;43:e331-3.

47. Dekker I, van der Leest M, van Rijk MC, Gerritsen WR, Arens AIJ. 68Ga-PSMA uptake in Angiolipoma. Clin Nucl Med. 2018;43:757.

48. Itabashi T, Emori M, Terashima Y. Hemangioma of the rib showing a relatively high 18 F-FDG uptake: a case report with a literature review. Acta Radiol Open. 2017;6:1-5.

49. Chaussé G, Laufer J, Abikhzer G, Probst S. Splenic hemangioma as a potential pitfall on PSMA-targeted 18F-DCFPyL PET/CT. Clin Nucl Med. 2019; 44:255-6.

50. Finberg $H$, Levin D. Angiolipoma: a rare benign soft tissue tumor with a malignant arteriographic appearance. Am J Roentgenol. 1977:128:697-8.

51. Artigas C, Otte F-X, Lemort M, van Velthoven R, Flamen P. Vertebral hemangioma mimicking bone metastasis in 68Ga_PSMA ligand PET/CT. Clin Nucl Med. 2017:42:368-70.

52. Jochumsen MR, Vendelbo MH, Høyer S, Bouchelouche K. Subcutaneous lobular capillary hemangioma on 68Ga-PSMA PET/CT. Clin Nucl Med. 2017; 42:e214-5.

53. Bhardwaj $H$, Stephens $M$, Bhatt $M$, Thomas PA. Prostate-specific membrane antigen PET/CT findings for hepatic hemangioma. Clin Nucl Med. 2016;41: 968-9.

54. Wang W, Tavora F, Sharma R, Eisenberger M, Netto GJ. PSMA expression in schwannoma: a potential clinical mimicker of metastatic prostate carcinoma. Urol Oncol Semin Orig InvestigElsevier Inc. 2009;27:525-8.

55. Rischpler C, Maurer T, Schwaiger M, Eiber M. Intense PSMA-expression using 68Ga-PSMA PET/CT in a paravertebral schwannoma mimicking prostate cancer metastasis. Eur J Nucl Med Mol Imaging. 2016:43:193-4.

56. Kanthan GL, Izard MA, Emmett L, Hsiao E, Schembri GP. Schwannoma showing avid uptake on 68Ga-PSMA-HBED-CC PET/CT. Clin Nucl Med. 2016; 41:703-4.

57. Shenderov E, Gorin MA, Kim S, Johnson PT, Allaf ME, Partin AW, et al. Diagnosing small bowel carcinoid tumor in a patient with oligometastatic prostate cancer imaged with PSMA-targeted [ 18 F] DCFPyL PET/CT: value of the PSMA-RADS-3D designation. Urol Case Rep. 2018;17:22-5.

58. Ost P, Bossi A, Decaestecker K, De Meerleer G, Giannarini G, Karnes RJ, et al. Metastasis-directed therapy of regional and distant recurrences after curative treatment of prostate cancer: a systematic review of the literature. Eur Urol. 2015;67:852-63.

59. Ruf J, Huber-Schumacher S, Stoykow C, Almanasreh N, Jilg C. Strong PSMA Radioligand uptake by rectal carcinoma. Clin Nucl Med. 2016;42:225-6.

60. Hangaard L, Jochumsen MR, Vendelbo MH, Bouchelouche K. Metastases from colorectal cancer avid on 68Ga-PSMA PET/CT. Clin Nucl Med. 2017:42: 532-3.

61. Kanthan GL, Coyle L, Kneebone A, Schembri GP, Hsiao E. Follicular lymphoma showing avid uptake on 68Ga PSMA-HBED-CC PET/CT. Clin Nucl Med. 2016:41:500-1.

62. Osman MM, Iravani A, Hicks RJ, Hofman MS. Detection of synchronous primary malignancies with $68 \mathrm{Ga}$-labeled prostate-specific membrane antigen PET/CT in patients with prostate cancer: frequency in 764 patients. $J$ Nucl Med. 2017:58:1938-42.

63. Chang SS, Reuter VE, Heston WD, Bander NH, Grauer LS, Gaudin PB. Five different anti-prostate-specific membrane antigen (PSMA) antibodies confirm PSMA expression in tumor-associated neovasculature. Cancer Res. 1999;59:3192-8.

64. Chang SS, Reuter VE, Heston WDW, Gaudin PB. Metastatic renal cell carcinoma neovasculature expresses prostate-specific membrane antigen. Urology. 2001:57:801-5.

65. Bychkov A, Vutrapongwatana U, Tepmongkol S, Keelawat S. PSMA expression by microvasculature of thyroid tumors - potential implications for PSMA theranostics. Sci RepSpringer US. 2017;7:1-11.

66. Siva S, Callahan J, Pryor D, Martin J, Lawrentschuk N, Hofman MS. Utility of $68 \mathrm{Ga}$ prostate specific membrane antigen - positron emission tomography in diagnosis and response assessment of recurrent renal cell carcinoma. J Med Imaging Radiat Oncol. 2017;61:372-8.

67. Verma P, Malhotra G, Goel A, Rakshit S, Chandak A, Chedda R, et al. Differential uptake of 68Ga-PSMA-HBED-CC (PSMA-11) in low-grade versus high-grade gliomas in treatment-naive patients. Clin Nucl Med. 2019;44: e318-22.

68. Taneja S, Taneja R, Kashyap V, Jha A, Jena A. Ga-PSMA uptake in hepatocellular carcinoma. Clin Nucl Med. 2016;42:69-70.

69. Haffner MC, Kronberger IE, Ross JS, Sheehan CE, Zitt M, Mühlmann G, et al Prostate-specific membrane antigen expression in the neovasculature of gastric and colorectal cancers. Hum PatholElsevier Inc. 2009:40:1754-61.

70. Salas Fragomeni RA, Amir T, Sheikhbahaei S, Harvey SC, Javadi MS, Solnes $\mathrm{LB}$, et al. Imaging of nonprostate cancers using PSMA-targeted radiotracers: rationale, current state of the field, and a call to arms. J Nucl Med. 2018;59: 871-7.

71. Rahbar K, Afshar-Oromieh A, Jadvar H, Ahmadzadehfar H. PSMA Theranostics: current status and future directions. Mol Imaging. 2018;17:1-9.

72. Rhee H, Blazak J, Tham CM, Ng KL, Shepherd B, Lawson M, et al. Pilot study: use of gallium-68 PSMA PET for detection of metastatic lesions in patients with renal tumour. EJNMMI Res. 2016;6:76.

73. Klein Nulent TJW, van Es RJJ, Krijger GC, de Bree R, Willems SM, de Keizer B. Prostate-specific membrane antigen PET imaging and immunohistochemistry in adenoid cystic carcinoma-a preliminary analysis. Eur J Nucl Med Mol Imaging. 2017:44:1614-21.

74. Sathekge M, Lengana T, Modiselle M, Vorster M, Zeevaart J, Maes A, et al. 68Ga-PSMA-HBED-CC PET imaging in breast carcinoma patients. Eur J Nucl Med Mol Imaging. 2017;44:689-94..

\section{Publisher's Note}

Springer Nature remains neutral with regard to jurisdictional claims in published maps and institutional affiliations.
Ready to submit your research? Choose BMC and benefit from:

- fast, convenient online submission

- thorough peer review by experienced researchers in your field

- rapid publication on acceptance

- support for research data, including large and complex data types

- gold Open Access which fosters wider collaboration and increased citations

- maximum visibility for your research: over $100 \mathrm{M}$ website views per year

At $\mathrm{BMC}$, research is always in progress.

Learn more biomedcentral.com/submissions 